\title{
Haplotype-Phased Genome Assembly of Virulent Phytophthora ramorum Isolate ND886 Facilitated by Long-Read Sequencing Reveals Effector Polymorphisms and Copy Number Variation
}

\author{
Mathu Malar C,, Jennifer D. Yuzon,, ${ }^{3,4}$ Subhadeep Das, ${ }^{1,2}$ Abhishek Das,, ${ }^{1,2}$ Arijit Panda, ${ }^{1,2}$ \\ Samrat Ghosh, ${ }^{1,2}$ Brett M. Tyler, ${ }^{5, \dagger}$ Takao Kasuga, ${ }^{3,4, \dagger}$ and Sucheta Tripathy ${ }^{1,2,+}$ \\ ${ }^{1}$ Computational Genomics Lab, Structural Biology and Bioinformatics Division, CSIR Indian Institute of Chemical Biology, \\ Kolkata, 700032, India \\ ${ }^{2}$ Academy of Scientific and Innovative Research (AcSIR), Ghaziabad, India \\ ${ }^{3}$ Department of Plant Pathology, University of California, Davis, CA, U.S.A. \\ ${ }^{4}$ USDA-ARS, Davis, CA, U.S.A. \\ ${ }^{5}$ Center for Genome Research and Biocomputing and Department of Botany and Plant Pathology, Oregon State University, \\ Corvallis, OR 97331-7303, U.S.A.
}

Accepted 7 February 2019.

\begin{abstract}
Phytophthora ramorum is a destructive pathogen that causes sudden oak death disease. The genome sequence of $P$. ramorum isolate Pr102 was previously produced, using Sanger reads, and contained $12 \mathrm{Mb}$ of gaps. However, isolate Pr102 had shown reduced aggressiveness and genome abnormalities. In order to produce an improved genome assembly for $P$. ramorum, we performed long-read sequencing of highly aggressive $P$. ramorum isolate CDFA1418886 (abbreviated as ND886). We generated a 60.5-Mb assembly of the ND886 genome using the Pacific Biosciences (PacBio) sequencing platform. The assembly includes 302 primary contigs $(60.2 \mathrm{Mb})$ and nine unplaced contigs $(265 \mathrm{~kb})$. Additionally, we found a 'highly repetitive' component from the PacBio unassembled unmapped reads containing tandem repeats that are not part of the $60.5-\mathrm{Mb}$ genome. The overall repeat content in the primary assembly was much higher than the Pr102 Sanger version (48 versus $29 \%$ ), indicating that the long reads have captured repetitive regions effectively. The 302 primary contigs were phased into 345 haplotype blocks and 222,892 phased variants, of which the longest phased block was $1,513,201$ bp with 7,265 phased variants. The improved phased assembly facilitated identification of 21 and 25 Crinkler effectors and 393 and 394 RXLR effector genes from two haplotypes. Of these, 24 and 25 RXLR effectors were newly predicted from haplotypes $A$ and $B$, respectively. In
\end{abstract}

Mathu Malar C and Jennifer D. Yuzon were equal contributors.

${ }^{\dagger}$ Corresponding authors: B. M. Tyler; Brett.Tyler@oregonstate.edu, T. Kasuga; tkasuga@ucdavis.edu, and

S. Tripathy; tsucheta@iicb.res.in or tsucheta@gmail.com

Funding: This work was funded by the Department of Science and Technology, Ministry of Science and Technology DST-Inspire AORC.

*The $\boldsymbol{e}$-Xtra logo stands for "electronic extra" and indicates that 12 supplementary figures, one supplementary table, and six supplementary dataset files are published online.

The author(s) declare no conflict of interest.

(c) 2019 The American Phytopathological Society addition, seven new paralogs of effector Avh207 were found in contig 54, not reported earlier. Comparison of the ND886 assembly with Pr102 V1 assembly suggests that several repeatrich smaller scaffolds within the Pr102 V1 assembly were possibly misassembled; these regions are fully encompassed now in ND886 contigs. Our analysis further reveals that Pr102 is a heterokaryon with multiple nuclear types in the sequences corresponding to contig 10 of ND886 assembly.

Keywords: genomics, Phytophthora diseases

Phytophthora ramorum is a devastating pathogen that infects a wide range of plant species and is invasive to Western Europe and North America. The NA1 lineage of P. ramorum is the causal agent of the sudden oak death epidemic in California that is killing members of family Fagaceae and a wide range of other plant families (Grünwald et al. 2008). To understand the genetic basis of pathogen behavior, the first $P$. ramorum draft genome, based on Sanger sequencing, was published in 2006, using isolate Pr102 from coast live oak (Quercus agrifolia) (Tyler et al. 2006). Genome analyses of Phytophthora spp. have shown that these pathogens secrete a diverse array of pathogenicity-related effector proteins (Jiang and Tyler 2012). The majority of effectors come from the RXLR (also called Avh) superfamily, which plays an important role in pathogenicity (Jiang et al. 2008). Genome architecture studies of Phytophthora infestans and Phytophthora sojae revealed that the effector genes of Phytophthora spp. are not randomly distributed in the genome (Haas et al. 2009; Tyler et al. 2006). Rather, the Phytophthora genome is arranged into regions with two distinct characteristics, namely, gene-dense regions interspersed with gene-sparse regions that contain the majority of effector genes (Raffaele and Kamoun 2012). The 2006 reference genome of $P$. ramorum has facilitated studies on epigenetic regulation of effector gene expression and genome plasticity (Elliott et al. 2018; Kasuga et al. 2012, 2016). These genomic studies suggest that a unique genomic architecture and mechanisms of genomic plasticity allow oomycetes such as $P$. ramorum to evolve diversified effector repertoires. 
For $P$. ramorum, however, the incompleteness of the Pr102 genome assembly posed several problems for comparing genomes of additional isolates for finer scale epidemiology and genetic studies. First, the 2006 assembly contained 2,576 scaffolds encompassing $12 \mathrm{Mb}$ of gaps, compared with the ten to 12 chromosomes of $P$. ramorum ( $n=10$ to 12 , based on cytological examination [Beattie 2009]). As a result of the gaps, the higher-level architecture of the pathogen genome was obscured, and potentially, many effector genes and repetitive elements were lost or their relative locations were unclear. Second, the haplotypes of $P$. ramorum, which is diploid, were not resolved. The 2006 genome assembly of $\operatorname{Pr} 102$ was a consensus comprised of a mosaic of haplotypes based on the most frequent sequence reads at each polymorphic site. This fact impedes analyses involving allelic variation and linkages between polymorphisms. Therefore, to provide a foundation for more detailed studies of pathogen evolution, we used Pacific Biosciences (PacBio) long-read sequencing technology to produce a more complete genome assembly with fewer gaps.

'Phasing' or the estimation of haplotypes can help distinguish the location of a polymorphism on a specific haplotype and identify other polymorphisms that are located close by on the same DNA strand. Improvements in long-read (multi-kilobase) sequencing technologies have enabled the rapid and costeffective assembly of large, complex genomes (Gnerre et al. 2011; Lam et al. 2012). Long-read sequencing technologies include polymerase chain reaction (PCR)-free methods to reduce PCR or GC bias (Rhoads and Au 2015) and to conserve the fidelity of alleles when assembling the genome. Single molecule real-time (SMRT) sequencing technology (English et al. 2012) produces multi-kilobase reads that enable production of genome assemblies with longer contigs that can effectively fill gaps, producing a less-fragmented assembly (Vij et al. 2016). The longer contigs enable haplotype phasing in diploids and polyploids in identifying individual alleles (Seo et al. 2016). Phasing provides valuable resources for studying allelic variation, linkage disequilibrium (LD), and larger-scale genetic variation.

Using PacBio SMRT sequencing technology, combined with a refined bioinformatics pipeline that includes the FALCON assembler (Chin et al. 2016), we have successfully filled most of the gaps and phased many regions of the genome of $P$. ramorum ND886. With the upgraded $P$. ramorum assembly, we were able to document a number of additional effector genes and transposable elements, many of which corresponded to gaps in the 2006 version of the assembly. The upgraded genome assembly also revealed numerous effector polymorphisms not evident in the earlier assembly. The upgraded $P$. ramorum genome assembly will enable a higher-resolution study of the underlying genetic variation in the virulence genes of $P$. ramorum. FALCON produced large primary contigs that could be directly used in haplotype resolution, aiding studies of effector heterozygosity and genome architecture.

\section{RESULTS}

\section{Preliminary sequencing results} for isolates ND886 and Pr102.

The objective was to improve the genome sequence of the sudden oak death pathogen $P$. ramorum. The isolate Pr102, used in the 2006 genome project, was originally isolated from coast live oak (Quercus agrifolia). This isolate shows reduced aggressiveness and growth rate (Elliott et al. 2018; Kasuga et al. 2012) and was suggested to be trisomic (Elliott et al. 2018; Kasuga et al. 2016). Flow cytometry analysis showed that the nuclear content of Pr102 was not stable (Supplementary Fig. S1). To understand the pathogenicity of $P$. ramorum at the genome level, an aggressive isolate carrying an intact genome was needed. A Californian standard isolate ND886 (CDFA1418886) derived from camellia $\times$ 'Coral Delight' showed normal aggressiveness and growth rate (Elliott et al. 2018; Kasuga et al. 2016). The nuclear content of ND886 was stable and consistent with the published value $(75 \mathrm{Mb})$ for an NA1 isolate (Vercauteren et al. 2011). Isolates ND886 and Pr102 were obtained from similar geographical locations (about $10 \mathrm{~km}$ apart) 10 to 20 years after the inferred introduction of the pathogenic clone to California. Therefore, we expected that the two isolates should be genetically similar, apart from the number of chromosomes. We sequenced ND886 using the PacBio (PacBio) P6-C4 chemistry, generating 402,170 reads at $70 \times$ with average read length of $10.5 \mathrm{~kb}$, longest read length of 50,801 bp, and median read length of 9,762 bp.

\section{Genome assembly of ND886 using FALCON assembler.}

FALCON assembler (Chin et al. 2016) was used to produce the main draft primary contigs. FALCON produces two types of contigs, namely primary contigs and associated contigs (which mainly contain regions with structural variants, allelic to the primary contigs). FALCON reduces assembly problems linked with heterozygocity and handles ploidy successfully, generating more contiguous contigs. Using this method, 302 primary contigs, totaling $60,221,882 \mathrm{bp}$ and having a contig $\mathrm{N}_{50}$ of $687,183 \mathrm{bp}$, were obtained. There were 397 associated contigs (most likely allelic to the primary contigs), comprising $18,850,872 \mathrm{bp}$ with an $\mathrm{N}_{50}$ of 55,130 bp (data available at EUmicrobeDB). All the PacBio raw reads were mapped back to the $60.2-\mathrm{Mb}$ primary contigs and $18 \mathrm{Mb}$ of associated contigs, resulting in a total of 13,300 unmapped reads. These unmapped reads were further assembled using Canu (Koren et al. 2017) (Table 1; Supplementary Fig. S2), resulting in nine contigs encompassing about $265 \mathrm{~kb}$ (smallest: 13,373 bp, largest:

Table 1. Assembly statistics of Phytophthora ramorum isolates ${ }^{\mathrm{a}}$

\begin{tabular}{|c|c|c|c|c|c|c|}
\hline Assembly name & Total contigs & Assembly size (in bp) & No. of genes & $\mathbf{N}_{50}$ & Total gaps & $\begin{array}{l}\text { No. BUSCO } \\
\text { COGs }\end{array}$ \\
\hline Pr102 V1 (Tyler et al. 2006) & 2,576 & $66.6 \mathrm{Mb}$ & 16,134 & $\begin{array}{l}\text { Scaffold: } 308,042 \mathrm{bp} \\
\text { Contig: } 47,500 \mathrm{bp}\end{array}$ & $12.2 \mathrm{Mb}$ & 230 \\
\hline $\begin{array}{l}\text { ND886 (primary contigs) }+ \\
\text { unplaced contigs }\end{array}$ & $302+9$ & $60,221,882+265,202$ & $12,337+42$ & $68,718,331,753$ & NA & $155+\mathrm{NA}$ \\
\hline ND886 (associated contigs) & 397 & $18,850,872$ & NA & 55,130 & NA & 66 \\
\hline $\begin{array}{l}\text { ND886 (consensus } \\
\text { haplotype A) }\end{array}$ & 302 & $60,284,349$ & 14,470 & 688,117 & NA & 231 \\
\hline $\begin{array}{l}\text { ND886 (consensus } \\
\text { haplotype B) }\end{array}$ & 302 & $60,285,334$ & 14,998 & 687,920 & NA & 231 \\
\hline $\begin{array}{l}\text { ND886 (highly repetitive } \\
\text { component) }\end{array}$ & 3,275 & $23,217,622^{b}$ & NA & 12,139 & NA & NA \\
\hline
\end{tabular}

\footnotetext{
${ }^{a}$ Genome assembly statistics of primary contigs, unplaced contigs, consensus haplotype assemblies, and highly repetitive component are shown here with total gaps present in assembly with number of genes predicted from assemblies. COGs = clusters of orthologous groups. NA = not available

$\mathrm{b}$ This is not an assembly. Most reads are singletons.
} 
$76,986 \mathrm{bp}, \mathrm{N}_{50}$ value: $31,825 \mathrm{bp}$ ), which are designated as unplaced contigs. These sequences were not incorporated into the initial FALCON assembly, presumably because of the presence of complex repetitive elements. Canu also produced a residue of 3,275 unassembled low-coverage sequences (3,254 singletons and 21 contigs) that were included in the analysis for checking for tandem repeats. Supplementary Dataset File 1 contains tandem repeats and the statistics information of 3,275 unassembled sequences with low coverage $(3,254$ of the reads were singletons, matching no other sequences) that were not part of the primary assembly, which we designated as the highly repetitive (HR) component. The HR component comprised a total length of $23,217,622 \mathrm{bp}$; however, most of this length was composed of unassembled singleton reads. The smallest sequence was $1,015 \mathrm{bp}$ and the largest sequence was $41,015 \mathrm{bp}$, with a $\mathrm{N}_{50}$ of $12,139 \mathrm{bp}$. Some of the most abundant repeat units in the HR component were $<10 \mathrm{bp}$ in size (Table 2). Tandem repeat units of $\geq 10$ with repeat numbers larger than 100 were also present in this assembly (Supplementary Fig. S3). These types of repeats are missing from the $P$. sojae assembly and are greatly reduced in the $P$. ramorum Pr102 Sanger assembly. Quality assessments of the primary assembly using QUAST compared with the older assembly are shown in Supplementary Fig. S4. Our transposon predictions from the phased consensus haplotypes of ND886 and Pr102 V1 identified a greater number of mobile elements from the PacBio-based assembly. In particular, a greater number of gypsy and, especially, copia elements were identified (Table 3).

Repeats from phased haplotypes and Pr102 V1 assembly are summarized in Supplementary Table S1.

Table 2. Abundant tandem repeats in the highly repetitive (HR) regions in Phytophthora ramorum ${ }^{\mathrm{a}}$

\begin{tabular}{lccc}
\hline $\begin{array}{l}\text { Predominant repeat sequence } \\
\text { in HR component of ND886 }\end{array}$ & $\begin{array}{c}\text { Unit } \\
\text { length } \\
\text { (bp) }\end{array}$ & $\begin{array}{c}\text { Number } \\
\text { in ND886 }\end{array}$ & $\begin{array}{c}\text { Comparison } \\
\text { with Pr102 } \\
\text { Phyra V1 }\end{array}$ \\
\hline GCGACCGTTG & 10 & 948 & No match \\
TCGCCAACGG & 10 & 806 & 8 \\
CGCCAACCCAT & 11 & 228 & No match \\
AACGGTCGCC & 10 & 195 & 8 \\
GTTGGCGATGG & 11 & 163 & No match \\
GGAGACCACGACGGGAGAC & 19 & 113 & No match \\
CTTGTTGATTAA & 12 & 72 & No match \\
\hline
\end{tabular}

${ }^{a}$ Most abundant tandem repeat elements from HR region in comparison with $P$. ramorum $\mathrm{V} 1$ are shown here.

\section{Genotype calling using Illumina reads.}

To correct errors in PacBio sequencing and to help identify heterozygous sites for haplotype phasing, we also sequenced the ND886 genome using the Illumina platform in June 2016 (55x), July $2017(63 \times)$, and August $2017(86 \times)$, resulting in three sequence libraries. Read depth was calculated using SAMtools (Li et al. 2009) to compute the depth at each position for each library. Libraries were aligned to the $60.2-\mathrm{Mb}$ primary contigs, generated from the FALCON assembler, using bwa aln (Li and Durbin 2009). The genotypes were called using SAMtools mpileup and Bcftools (Li 2011). Genotypes at heterozygous loci that occurred in at least two libraries were kept for use for phasing. Genotypes at heterozygous loci with two alternative alleles (e.g., 1/2) were filtered, using bcftools ( $\mathrm{Li}$ 2011), because we were mapping ND886 to itself. Therefore, only the allele present in the genome assembly and one additional allele (genotype 0/1 in the VCF file) should be present. Altogether 223,382 heterozygous single nucleotide polymorphisms (SNPs) were cataloged as a basis for haplotype estimation.

\section{Haplotype phasing by combining Illumina genotypes with PacBio read data.}

The reconstruction of haplotypes can capture genetic variants that can be missed in unphased genomes (e.g., indels, structural variants, and gene conversion). The assembled primary contigs of ND886 were used for read-based phasing with WhatsHap, using vcf files containing ND886 Illumina data as well as BAM files from the PacBio alignment (Garg et al. 2016), to produce a haplotype-phased diploid assembly. The genetic phasing resulted in two haplotypes (A and B) for heterozygous regions in the genome. Haplotype A of one contig and haplotype A of another contig are not necessarily physically linked or derived from the same parent. Rather, phased sequences of contigs were randomly assigned to haplotype A or B. After genotype calling using Illumina reads, the haplotype phasing of ND886 identified 223,294 heterozygous variants. Of these, 222,892 variants were phased into 345 blocks, encompassing $54 \mathrm{Mb}$ of the genome assembly. $P$. ramorum ND886 has, on average, 3.7 heterozygous SNPs for every $1 \mathrm{~kb}$, which is lower than $P$. capsici (23.4 single nucleotide variants [SNVs] per kilobase) and Phytophthora tropicalis (51.7 SNVs per kilobase) (Lamour et al. 2012) but higher than P. infestans (0.65 SNVs per kilobase) (Raffaele et al. 2010). The largest 25 scaffolds (encompassing 25,093,049 bp), showing phased and unphased SNPs, are plotted in Figure 1A, which indicates that the majority of the genome is phased (gray regions), with only a few regions unphased due to the low levels of heterozygous SNP density.

Table 3. Transposon elements identified in assemblies ${ }^{\mathrm{a}}$

\begin{tabular}{|c|c|c|c|c|}
\hline Transposable element & Transposon type & Pr102 V1 assembly & Haplotype A consensus & Haplotype B consensus \\
\hline Cacta & DNA transposons & 9 (912 bp) & $18(1,806 \mathrm{bp})$ & $18(1,812$ bp $)$ \\
\hline Crypton & DNA transposons & $224(114,156 \mathrm{bp})$ & $196(104,298 \mathrm{bp})$ & 197 (104,649 bp) \\
\hline DDE motif & Unknown & $250(123,543 \mathrm{bp})$ & $216(112,920 \mathrm{bp})$ & $214(113,070 \mathrm{bp})$ \\
\hline Gypsy & LTR retroelements & $1,992(1,441,695 \mathrm{bp})$ & $2,319(1,918,386 \mathrm{bp})$ & $2,315(1,909,431 \mathrm{bp})$ \\
\hline hAT & DNA elements & $117(70,713 \mathrm{bp})$ & $93(63,270 \mathrm{bp})$ & $92(65,871 \mathrm{bp})$ \\
\hline Helitron ORF & DNA transposons & $246(151,206 \mathrm{bp})$ & $223(183,378 \mathrm{bp})$ & $231(185,316 \mathrm{bp})$ \\
\hline ISC & Unknown & $34(12,459 \mathrm{bp})$ & $26(9,255 \mathrm{bp})$ & $25(9,009 \mathrm{bp})$ \\
\hline LINE & Non-LTR elements & $135(140,301 \mathrm{bp})$ & $193(202,137 \mathrm{bp})$ & $199(214,983 \mathrm{bp})$ \\
\hline ltr_Roo & Unknown & $44(33,753 \mathrm{bp})$ & $19(14,427 \mathrm{bp})$ & $18(11,220 \mathrm{bp})$ \\
\hline Mariner & DNA elements & $174(75,465 \mathrm{bp})$ & $178(77,067 \mathrm{bp})$ & 177 (77,016 bp) \\
\hline Mariner_ant & DNA elements & $92(40,464 \mathrm{bp})$ & $93(43,632 \mathrm{bp})$ & 94 (43,707 bp) \\
\hline MuDR_A_B & Uknown & $75(36,210 \mathrm{bp})$ & $56(33,315 \mathrm{bp})$ & 57 (32,862 bp) \\
\hline Piggybac & DNA elements & $258(139,008 \mathrm{bp})$ & 237 (145,896 bp) & $234(145,089$ bp $)$ \\
\hline Ty_copia & LTR retroelements & $1,346(964,962 \mathrm{bp})$ & $4,402(3,783,141 \mathrm{bp})$ & $4,406(3,796,428 \mathrm{bp})$ \\
\hline
\end{tabular}

a $\mathrm{ORF}=$ open reading frame; ISC = insertion sequences encoding Cas9 homolog family; LTR = long terminal repeat; LINE = long interspersed nuclear elements. 
The average number of variants per block was 646 and the median block length was 44,513 bp (EUmicrobeDB). The phased haplotypes were polished using the ND886 Illumina reads to reduce the indel errors from the phased assemblies. The polished haplotypes were used for downstream analysis.

\section{Chromosome copy number variation $(\mathrm{CCNV})$ and relationships between haplotype blocks.}

In order to examine CCNV across isolates, a read depth comparison was done using TitanCNA (Ha et al. 2014). All isolates belonging to the $P$. ramorum NA1 lineage are assumed to be clones because a population comparison of $P$. ramorum NA1 isolates estimated that the pathogen diverged 30 to 120 years ago (unpublished) and there are only 716 SNVs compared with 17,056,937 heterozygous sites after filtering (only $0.004 \%$ SNP variation [unpublished]). Illumina genome sequence data were generated for Pr102 as well as ND886. The Pr102 and ND886 read-depth ratios for each heterozygous SNPs were mapped to the ND886 haplotype-phased assembly and to the previous Pr102 V1 Sanger assembly for this analysis. We note that Pr102 may not be in the same phase as ND886 at every site, due to possible mitotic crossing over. Figure 1B shows the CCNV results mapped onto the first $50 \mathrm{Mb}$ of the Pr102 V1 assembly (the largest 53 scaffolds) and onto the first $30.3 \mathrm{Mb}$ of the ND886 2018 phased haplotypes (largest 32 scaffolds); mapping to the full set of scaffolds is shown in Supplementary Figures S5 and S6. CCNV was detected in sequences that mapped to scaffolds 29, 34, and 46 of the Pr102 V1 assembly and that mapped to contig 10 of the ND886 phased haplotype assembly. Additional regions of CCNV were observed in Pr102 sequences corresponding to contigs $43,44,52,66$, and 69 of the ND886 phased assembly (Table 4).
The levels of CCNV revealed by TitanCNA did not match a simple 2:1 versus $1: 1$ ratio for any of the contigs; those ratios would be expected if the relevant contigs were associated with a trisomic chromosome in Pr102. The log ratios between the Pr102 and ND886 read coverage of haplotypes A and B of contig 10 were 0.24 and 0.86 (1.18:1 and 1.81:1 ratios), respectively. To test if the ratios for haplotypes A and B were significantly different from $1: 1$, a Welch's $t$ test on the ranked data of the $\log$ ratios was performed. Log ratios for haplotype A of contig 10 were compared with those of haplotype A of contig 9 , which average 0.03 (i.e., about a 1:1 ratio). The difference in the means of the ranked data between contig $10(0.24)$ and contig $9(0.03)$ had a $P$ value less than $2.2 \times 10^{-16}$. The $\log$ ratios for haplotype $\mathrm{B}$ of contig 10 were also compared with those for haplotype $\mathrm{B}$ of contig 9 . The difference in means between the contigs ( 0.86 compared with 0.03$)$ after ranking the $\log$ ratios also had a $P$ value less than $2.2 \times 10^{-16}$. To test if the contig $10 \log$ ratio for haplotype $\mathrm{B}(0.86)$ was significantly different from 1.0 (corresponding to a 2:1 ratio), we compared the $\log$ ratio distribution of haplotype $\mathrm{B}$ contig 10 with a theoretical contig having log ratio distribution with a median of 1 , corresponding to a 2:1 copy number ratio (haplotype B contig 9 with the median adjusted to 1.02 , producing a mean of 1.03). The $P$ value was less than $2.2 \times 10^{-16}$, which indicates that the observed CCNV of haplotype B contig 10 does not represent a $2: 1$ ratio. Since the CCNV ratios for haplotype A are significantly higher than $1: 1$ and the ratios for haplotype B are significantly lower than 2:1, we infer that Pr102 may be a heterokaryon with multiple nuclear types in the sequences corresponding to contig 10 .

Several contigs showed similar patterns of CCNV in the corresponding Pr102 sequences (Table 4). For haplotype A, the first half of contig 44 and contig 52 and the last half of contig
A

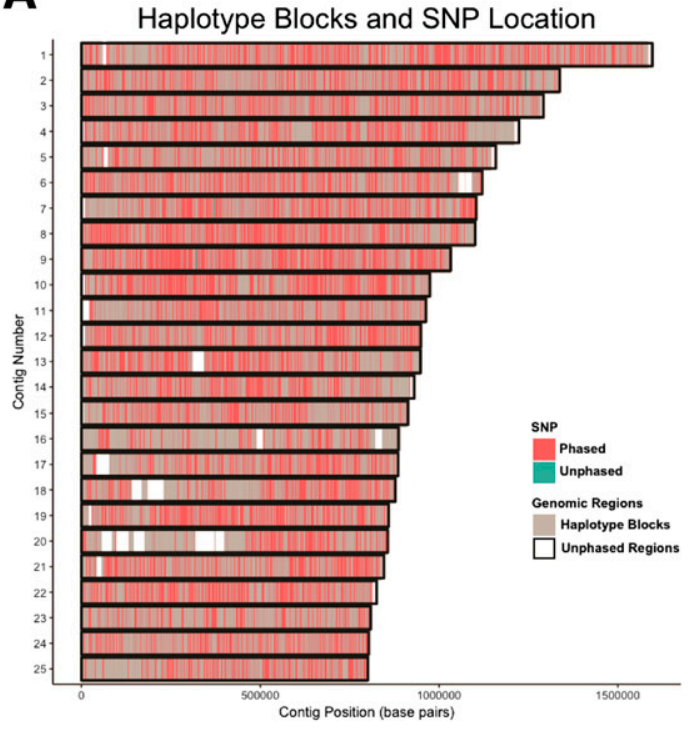

B
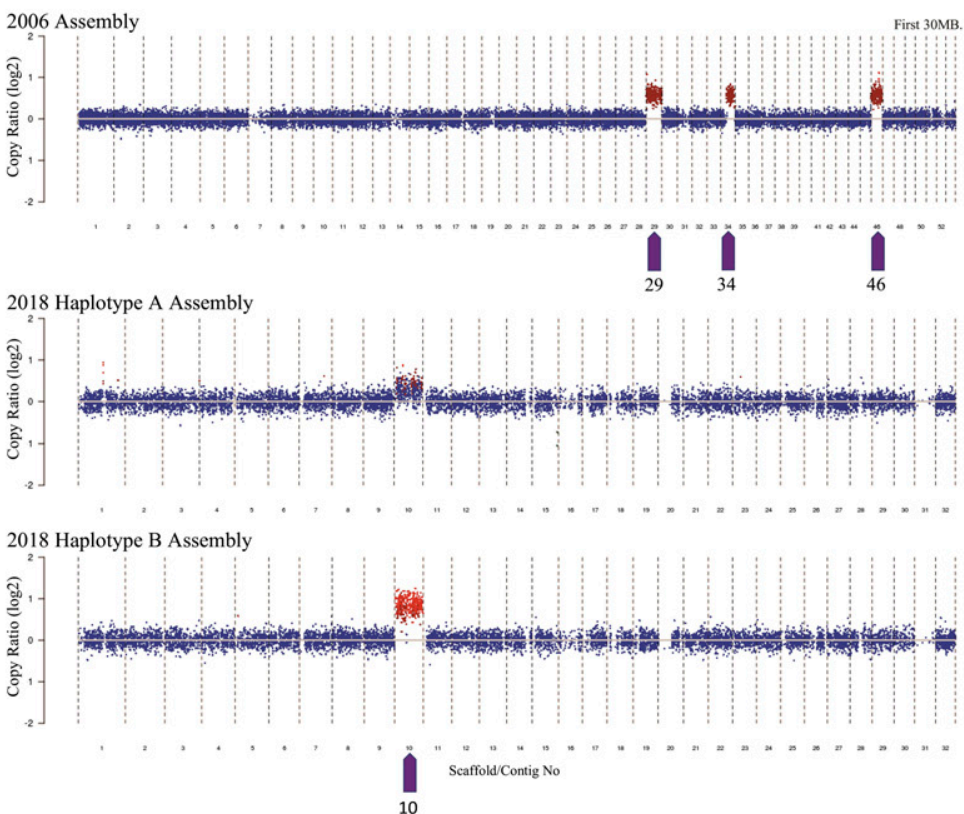

Fig. 1. Haplotype blocks containing single nucleotide polymorphisms (SNPs) and chromosome copy number variations (CCNVs) along with the phase information. A, The 25 largest contigs of the Phytophthora ramorum ND886 assembly are shown with the positions of SNPs along the length of the scaffolds. Unphased SNPs are represented as blue lines and phased SNPs are represented as red lines. The gray shaded regions in the plot represent the haplotype blocks and white represents regions of the genome that could not be passed due to lack of SNPs. B, CCNVs in Pr102 are mapped onto Pr102 and ND886 assemblies. The $y$ axis is the log ratio of normalized read depths between Pr102 and ND886 for each heterozygous locus. Red and blue datapoints indicate, respectively, significant or nonsignificant read depth ratio differences between isolates. The top graph shows combined log ratios for both haplotypes mapped onto the Pr102 V1 unphased genome assembly. Lower graphs show individual log ratios for each haplotype (middle, haplotype A; bottom, haplotype B) mapped to the respective haplotypes of the ND886 phased assembly. Purple arrows refer to scaffolds or contigs that have CCNV in Pr102. The first $50 \mathrm{Mb}$ of the Pr102 assembly (53 scaffolds) and the first $30 \mathrm{Mb}$ of the ND886 assembly (largest 32 scaffolds) are shown. 
66, contig 110, and contig 112 showed a log ratio between Pr102 and ND886 sequences of 0.69 to 0.88 , significantly different than the control $(P<2.2 \mathrm{e}-16)$. For haplotype $\mathrm{B}$, contig 43 and the last half of contig 44 showed a log ratio between Pr102 and ND886 sequences of 0.80 to 0.84 , significantly different than the control $(P<2.2 \mathrm{e}-16)$. Contig 69 contained a number of datapoints showing potential CCNV, but the aggregate CCNV for the contig was not statistically significant (Table 4). Only the second half of contig 66 shows CCNV of 1.61 and, thus, may represent a partial duplication. Contig 44 is comprised of two haplotype blocks that were arbitrarily assigned as haplotypes A and B for the first and second blocks, respectively. Haplotype A of the first half and haplotype B of the second half show approximately $1.8 \mathrm{CCNV}$. Haplotype B of the first half and haplotype A of the second half show approximately $1.2 \mathrm{CCNV}$. This strongly suggests physical linkage of haplotype $\mathrm{A}$ of the first half and haplotype $\mathrm{B}$ of the second half of contig 44 in Pr102, since both show 1.8 CCNV. Correspondingly, a physical linkage between haplotype $\mathrm{B}$ of the first half and haplotype A of the second half appear physically linked in $\operatorname{Pr} 102$.

The regions showing CCNV in Pr102 in comparison with the ND886 genome showed colinearity between the Pr102 and ND886 assemblies. The colinearity analysis revealed that contig 10 of ND886 was collinear with scaffolds 297, 68, 29, and 286; contig 43 was collinear with scaffolds 121,114 , and 46 ; contig 44 was collinear with scaffolds 94,75 , and 82 ; contig 52 was collinear with scaffolds 74 and 205; contig 66 was collinear with scaffolds $63,184,160$, and 94; contig 110 was collinear with scaffold 41 ; and contig 112 was collinear scaffolds 82 and 170. These regions are plotted in Supplementary Fig. S7.

\section{Prediction of genes in the phased haplotype assembly.}

Gene prediction was performed on the unpolished genome assembly (primary contigs) and on the polished haplotype-phased consensus assembly (A and B), using Scipio (Keller et al. 2008) and Augustus. A total of 12,337 genes were predicted in the unpolished primary contigs, and 42 in the $265 \mathrm{~kb}$ of unplaced contigs. A total of 14,470 and 14,998 genes were predicted in the polished consensus haplotypes $\mathrm{A}$ and $\mathrm{B}$ (Table 1), respectively; these numbers include the $6 \mathrm{Mb}$ of unphased sequences. The differences in the three gene numbers reflect the presence of indel sequencing errors from the PacBio sequencing that were partially but not fully corrected by the polishing process.

From our analysis, contig $1(1,595,961 \mathrm{bp})$ contained the longest haplotype block (at 1,513,201 bp in size) and contained 7,265 phased variants spanning more than 420 protein coding genes. Our annotation of genes within those blocks identified Pfam domains such as reverse transcription, short chain dehydrogenase, pectate lyase, zinc fingers, WD repeat domains, annexin beta-glucan synthases, nucleases, hydrolases, proteases, helicases, potassium channel proteins, and several RXLR effectors (Supplementary Dataset File 2). In addition, contigs 20, 31, and 77 each contained six large haplotype-phased blocks. By using oomycete repeat sequence libraries from Repeat Masker 4.0.6 (Smit et al. 2016), we were able to identify repetitive elements comprising $48.11 \%$ of each haplotype.

\section{Gene space assessment of assemblies.}

To evaluate the completeness of the assembled genomes, orthologous genes (clusters of orthologous groups [COGs]) of stramenopiles were checked in the assembled genomes, using BUSCO V2 (Simão et al. 2015). The stramenopile core orthologs consisting of 234 COGs were used for the assessment. We found 230 nonredundant COGs in the $P$. ramorum Sanger assembly and the same number of COGs (230) was found in the P. sojae V5 (Panda et al. 2018) assembly. From our phased consensus haplotypes (haplotypes A and B), we identified a total of 231 COGs with only three stramenopile COG genes missing from each of the two haplotypes. Six orthologs detected by BUSCO were duplicated in both haplotypes. Gene models from haplotypes A and B were mapped to the Pr102 gene models using blat, which found that 13,085 and 14,902 gene models mapped to Pr102 gene models.

\section{New effector genes were identified, from the improved haplotype consensus assembly of ND886, using modified prediction methods.}

RXLR (Avh) gene families are highly evolving genes (Goss et al. 2013). In the Pr102 V1 assembly, 370 RXLR effectors were predicted using a modified protocol as described below (Supplementary Fig. S8). This includes 224 effectors in each haplotype nearly identical (99 to $100 \%$ identity at the nucleotide level) to those predicted in Pr102 V1. Avh172 alleles showing 149 and 151 amino acid identities are shown in Supplementary Figure S9 and Supplementary Dataset File 3. In addition, there were 14 newly identified paralogs in each of the ND886 haplotypes, corresponding to eight effector genes in the Pr102 V1 assembly. There were also 131 RXLR genes exhibiting indels relative to $\operatorname{Pr} 102 \mathrm{~V} 1$, including three newly identified paralogs in both the consensus haplotypes. These effectors have either sequence gaps in one of the two haplotypes leading to frame-shift and stop codons or missing parts in the $5^{\prime}$ end (Avh 271), when compared with their Pr102 counterpart. Effector Avh343 from two ND886 alleles showing an

Table 4. Ranked Welch's $t$ test of Pr102 chromosome copy number variations (CCNVs) compared with a 1:1 and a 2:1 read depth ratio ${ }^{\text {a }}$

\begin{tabular}{|c|c|c|c|c|c|c|c|c|}
\hline \multirow[b]{3}{*}{ Contig } & \multirow{2}{*}{\multicolumn{2}{|c|}{$\begin{array}{c}\text { Mean read depth } \\
\text { ratio }^{\text {b }}\end{array}$}} & \multirow{2}{*}{\multicolumn{2}{|c|}{$\begin{array}{c}\text { Mean read depth } \\
\log _{2} \text { ratio }\end{array}$}} & \multicolumn{4}{|c|}{$P$ value } \\
\hline & & & & & \multirow{2}{*}{$\underset{1: 1}{\text { Hap } 1}$} & \multirow{2}{*}{$\underset{2: 1}{\text { Hap } 1}$} & \multirow{2}{*}{$\begin{array}{c}\text { Hap } 2 \\
1: 1\end{array}$} & \multirow{2}{*}{$\underset{2: 1}{\text { Hap }} 2$} \\
\hline & Hap 1 & Hap 2 & Hap 1 & Hap 2 & & & & \\
\hline 10 & 1.82 & 1.18 & 0.86 & 0.24 & $<2.2 \mathrm{e}-16$ & $<2.2 \mathrm{e}-16$ & $2.2 \mathrm{e}-16$ & $<2.2 \mathrm{e}-16$ \\
\hline 43 & 1.74 & 1.21 & 0.80 & 0.28 & $<2.2 \mathrm{e}-16$ & $<2.2 \mathrm{e}-16$ & $<2.2 \mathrm{e}-16$ & $<2.2 \mathrm{e}-16$ \\
\hline 44 & 1.73 & 1.17 & 0.79 & 0.23 & $<2.2 \mathrm{e}-16$ & $<2.2 \mathrm{e}-16$ & $1.9 \mathrm{e}-14$ & $<2.2 \mathrm{e}-16$ \\
\hline 44 & 1.79 & 1.14 & 0.84 & 0.19 & $<2.2 \mathrm{e}-16$ & $<2.2 \mathrm{e}-16$ & $2.2 \mathrm{e}-16$ & $<2.2 \mathrm{e}-16$ \\
\hline 52 & 1.78 & 1.22 & 0.83 & 0.29 & $<2.2 \mathrm{e}-16$ & $2.4 \mathrm{e}-14$ & $<2.2 \mathrm{e}-16$ & $<2.2 \mathrm{e}-16$ \\
\hline 66 & 0.97 & 0.96 & -0.05 & -0.06 & 0.02 & $<2.2 \mathrm{e}-16$ & $1.0 \mathrm{e}-3$ & $<2.2 \mathrm{e}-16$ \\
\hline 66 & 1.61 & 1.04 & 0.69 & 0.05 & $<2.2 \mathrm{e}-16$ & $<2.2 \mathrm{e}-16$ & 0.01 & $<2.2 \mathrm{e}-16$ \\
\hline 110 & 1.65 & 1.28 & 0.72 & 0.36 & $<2.2 \mathrm{e}-16$ & $1.1 \mathrm{e}-07$ & $2.6 \mathrm{e}-07$ & $<2.2 \mathrm{e}-16$ \\
\hline 112 & 1.84 & 1.14 & 0.88 & 0.19 & $<2.2 \mathrm{e}-16$ & $5.1 \mathrm{e}-08$ & $1.5 e-10$ & $<2.2 \mathrm{e}-16$ \\
\hline
\end{tabular}

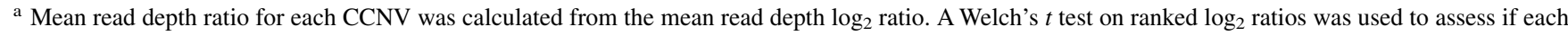
CCNV was significantly different than a $1: 1$ or $2: 1$ read depth ratio.

${ }^{\mathrm{b}}$ For each haplotype block, A and B were initially assigned arbitrarily. Haplotypes were then rearranged into two columns, haplotype 1 and 2, according to their physical linkage indicated by CCNV analysis. Bold indicate a CCNV is from haplotype B; italics indicate the CCNV is from haplotype A. 
indel are compared in Supplementary Fig. S10. We found 24 new RXLR effector genes in haplotype A and 25 in haplotype $\mathrm{B}$, representing 24 near-identical allele pairs in haplotypes $\mathrm{A}$ and B. However, there were five RXLR effector genes (Avh269, Avh299, Avh9, Avh336, Avh202) missing from both the ND886 haplotypes that were present in the Pr102 V1 assembly (Jiang et al. 2008; Tyler et al. 2006). These effectors are marked as missing due to the presence of indels. Avh207 had seven paralogs that were newly identified in the ND886 haplotypephased consensus assembly, in contigs 54 and 172 (Fig. 2). From our prediction, large numbers of effector genes were identified in contig $31(n=23)$ and in contig $50(n=24)$. Within the family of RXLR effectors, a subset of effectors has W and Y sequence motifs in the C-terminal domain that can be encoded by divergent amino acid sequences. Our initial hidden Markov model (HMM) search of all open reading frames with the WY functional motif identified 286 predicted proteins having WY motifs, and 176 of these had predicted signal peptides (Supplementary Dataset File 4).

In addition to the RXLR effectors, Phytophthora species secrete another group of effectors belonging to the Crinkler (CRN) family. The CRN effectors are characterized by a

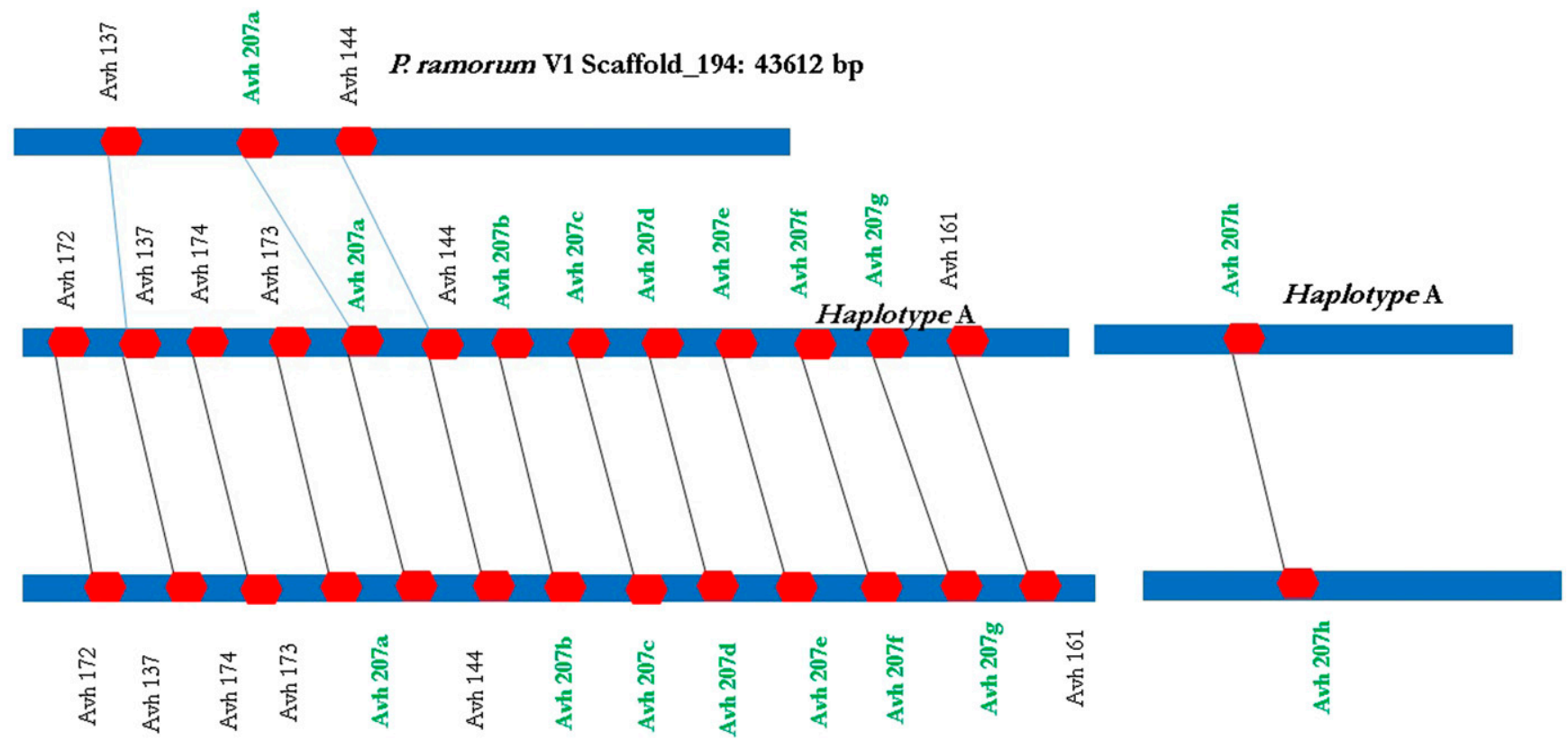

\section{Contig_54 Haplotype B Length $=388475$ bp}

\section{Contig_172 Haplotype B Length = 38131 bp}

Fig. 2. Phytophthora ramorum V1 containing one copy of Avh 207 from scaffold 194 is shown in the upper panel. The haplotype-phased assembly representing contig 54 and contig 172 from both the haplotypes depict the presence of multiple paralogs of Avh 207. The rectangular bar represents the contigs, the hexagons represent the $A v h$ genes from the contigs, and the black lines indicate the shared presence of these $R X L R$ genes in haplotypes A and B. The spaces and distances between the genes were drawn arbitrarily.

A Phytophthora ramorum ND886 consensus haplotype A

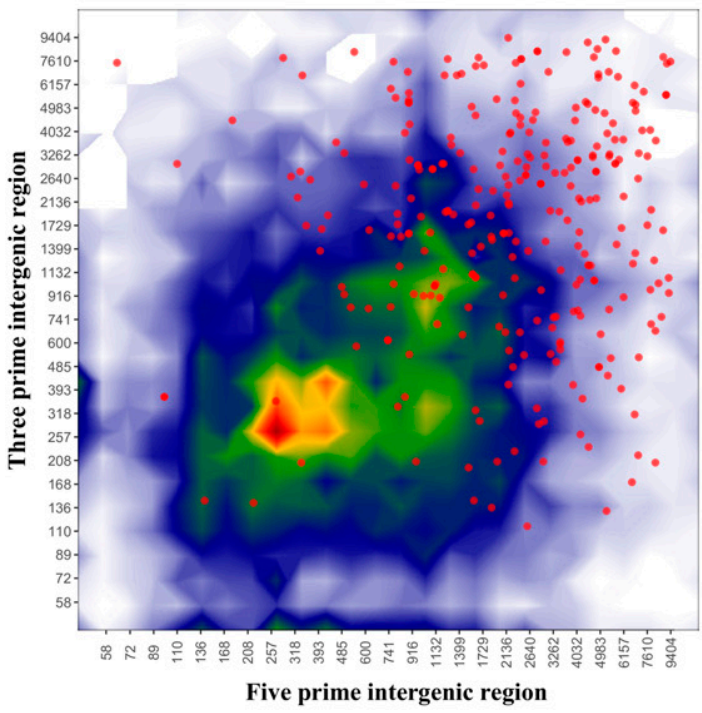

B Phytophthora ramorum ND886 consensus haplotype B

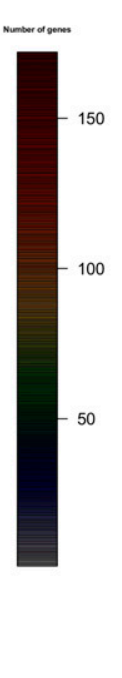

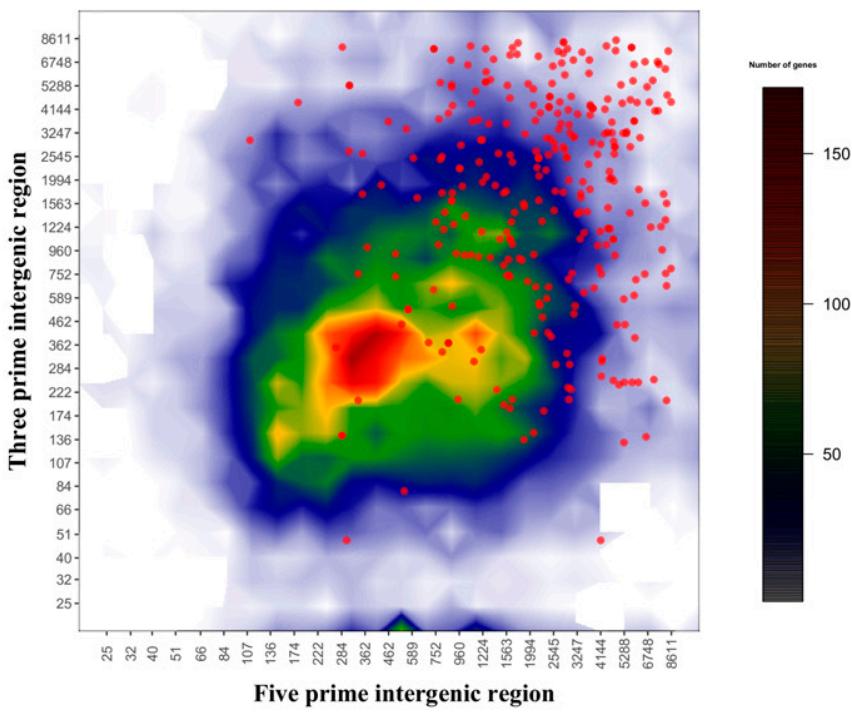

Fig. 3. The haplotype-phased Phytophthora ramorum ND886 genome shows the organization of the 'bi-partite' genome. A, Phased haplotype A. B, Phased haplotype B. Bright clusters in the heatmaps represent the gene-rich regions with fewer repeats. Circular dots indicate the CRN and RXLR effector genes. These genes are mainly found in the gene-sparse, repeat-rich regions. 
conserved N-terminal LXLFLAK motif, DWL domain, and diverse C-terminal effector domains (Haas et al. 2009; Schornack et al. 2010; Stam et al. 2013; Yin et al. 2017). The previous genome analyses of $P$. ramorum identified about $19 C R N$ genes (Haas et al. 2009). We predicted 21 and $25 C R N$ effector genes from the consensus haplotypes $\mathrm{A}$ and $\mathrm{B}$, using the methods described by Yin et al. (2017), out of which all 21 from haplotype A have an allelic complement in haplotype B. Approximately $50 \%$ of the CRN effectors predicted by our method did not have signal peptides that could be recognized by SignalP 3.0 (Käll et al. 2004). From the predicted CRN effector genes, 15 were polymorphic between ND886 haplotypes.

\section{Analysis of virulence-associated genes}

in carbohydrate-active enzyme (CAZy) families.

Proteins containing carbohydrate-binding modules have been reported as one family of virulence factors in plant pathogenic oomycetes (Brouwer et al. 2014). CAZys encoded in the $P$. ramorum ND886 and $P$. sojae genomes were predicted from the dbCAN database (Yin et al. 2012). A total of 107 CAZy families, containing 554 protein sequences, were predicted in $P$. ramorum ND886 haplotype A, and a total of 106 CAZy families containing 557 protein sequences were predicted in consensus haplotype B. A total of $105 \mathrm{CAZy}$ families were common to the two haplotypes, and there were five unique CAZy family members containing five proteins in haplotype A and four unique CAZy family members containing four protein sequences in haplotype B. In contrast, 85 families with 427 protein sequences were predicted in P. sojaeV5 (Panda et al. 2018). The most predominant families were found to be PL3 (polysaccharide lyase family 3), glycosyl transferases GT71 (glycosyl transferase), and glycosyl hydrolases (GH17) (Supplementary Dataset File 5). Of the CAZy proteins predicted to be potentially involved in pathogen-host interactions, there were 554 and 557 from ND886 phased consensus haplotypes A and $\mathrm{B}$, respectively, and 412 from $P$. sojae V5.

\section{A haplotype-phased $P$. ramorum ND886 genome confirms bipartite architecture.}

A phased genome is a more accurate representation of genetic diversity as opposed to the current conventional Phytophthora genome assemblies in which alleles of both haplotypes are conflated into chimeric consensus regions in the genome, which affects downstream analyses of genome evolution. Therefore, we used the phased ND886 genome to revisit the arrangement of the genome into conserved gene-rich regions and rapidly evolving gene-sparse regions (Baxter et al. 2010; Haas et al. 2009; Jiang et al. 2013; Tyler et al. 2006), commonly referred to as the 'two-speed genome' (Dong et al. 2015; Raffaele and Kamoun 2012). Thus, arrangement is observed in many filamentous pathogens and especially in oomycete pathogens (Dong et al. 2015; Haas et al. 2009; Raffaele and Kamoun 2012; Tyler et al. 2006). The criteria for evaluating bipartite genome architecture (i.e., two-speed genome) are based on the distributions of repetitive elements and effector genes and the colocalization of effectors in gene sparse regions. We tested whether the distribution of effector genes in $P$. ramorum was also associated with genomic compartments of low gene density and elevated repetitive elements, using the two-dimensional data binning approach (Saunders et al. 2014). As has been observed in several fungal and oomycete pathogens, the genome architecture of $P$. ramorum was confirmed to conform to a bipartite genome arrangement. First, the haplotype-phased consensus assembly of approximately 62.5 Mbp was rich (48.11\%) in repetitive elements. Second, the assembly contains a large repertoire of effector genes (393 in A and 394 in B haplotypes). Third, of those RXLR effector genes,
295 and 289 (in haplotypes A and B, respectively) overlapped with the repeat-rich regions within a range of $2 \mathrm{~kb}$, and 21 and $25 C R N$ effector genes were predicted from the two haplotypes. Of those, 14 and $17 C R N$ effector genes from haplotypes $\mathrm{A}$ and $\mathrm{B}$, respectively, also overlapped with repeat-rich regions within a span of $2 \mathrm{~kb}$. Fourth, the haplotype-phased genome map represented in Figure 3 clearly indicates that the effector genes are clustered in the gene-sparse regions of the genome. The mean and median values of intergenic distances for the haplotypes are provided in (Table 5). The mean $5^{\prime}$ and $3^{\prime}$ intergenic regions for the RXLR and CRN genes was four- to fivefold greater than the means for all genes.

\section{Nonsynonymous (dN) and synonymous (dS) substitution rates of effector genes.}

The RXLR genes from Pr102 V1 and haplotypes of ND886 were compared in order to compute the dS versus dN substitution rates. When multiple effectors were $100 \%$ identical, only one representative was included in the analysis. In order to identify orthologs, Blast searches were initially done on all versus all the RXLR genes for haplotype A versus Pr102 V1 and haplotype B versus Pr102 V1 comprising a total of around 500 genes for each search. Subsequently OrthoMCL (Li et al. 2003) was run using the blast output as input. There were 120 clusters encompassing haplotype A and Pr102 V1 plus 100 singletons. Of the 120 clusters, 58 had more than three members accounting for 266 of the total 500 genes (52\%) that were subsequently used for $\mathrm{dN}$ and $\mathrm{dS}$ calculations (Supplementary Dataset File 6). For haplotype B, there were a total of 120 clusters, of which only 68 clusters (accounting for 268 of 500 genes, 52\%) had more than three members and were used for $\mathrm{dN}$ and $\mathrm{dS}$ calculations. The number of singletons for haplotype $\mathrm{B}$ was 106 . We tested for statistically significant evidence for positive $(\mathrm{dN}>\mathrm{dS})$ and negative $(\mathrm{dN}<\mathrm{dS})$ selection affecting RXLR genes, using the PAML Yn00 package (Yang 1997) together with a multiple test correction (Benjamini and Hochberg 1995). However, no pairs showed statistically significant evidence for positive or negative selection.

\section{DISCUSSION}

\section{Overall PacBio assembled contigs are longer with greater repeat content.}

Assembly of heterozygous genomes has always been a challenge, especially when they are repeat-rich. In this study, PacBio genomic long reads were used to assemble the genome of $P$. ramorum isolate ND886, to produce a better draft contig assembly of $60.5 \mathrm{Mb}$ compared with the previously published Sanger assembly of the genome of isolate Pr102. FALCON (Chin et al. 2016) produced a $60.2 \mathrm{Mb}$ primary assembly

Table 5. Statistics of intergenic distances ${ }^{\mathrm{a}}$

\begin{tabular}{lcc}
\hline Intergenic distance values & $\begin{array}{c}\text { Consensus } \\
\text { haplotype A }\end{array}$ & $\begin{array}{c}\text { Consensus } \\
\text { haplotype B }\end{array}$ \\
\hline Mean 5' all genes & 1,151 & 1,176 \\
Mean 3' all genes & 990 & 943 \\
Median 5' all genes & 569 & 559 \\
Median 3' all genes & 405 & 366 \\
Mean 5' effector genes & 4,638 & 4,263 \\
Mean 3' effector genes & 4,753 & 4,002 \\
Median 5' effector genes & 3,007 & 2,837 \\
Median 3' effector genes & 2,703 & 2,518 \\
\hline
\end{tabular}

a Mean and median intergenic distances for all genes along with RXLR and Crinkler effector genes were calculated for the consensus phased haplotypes. 
(p-contigs) consisting of 302 contigs plus $265 \mathrm{~kb}$ of unplaced contigs. The earlier assembly (Pr102 V1) was produced exclusively using Sanger reads and the sequence content of the assembly was $54.4 \mathrm{Mb}$, excluding $12.2 \mathrm{Mb}$ of gaps within the 66.6 Mb assembly (Tyler et al. 2006). The ND886 primary contig assembly had a substantial increase in repeat content compared with the Pr102 V1 assembly; the repeat percentages were $48 \%$ (of p-contigs) and $29 \%$ in Pr102 V1 assembly, respectively, comprising $29.0 \mathrm{Mb}$ of the ND886 assembly and $15.8 \mathrm{Mb}$ of the Pr102 assembly. The difference in repeat content $(13.2 \mathrm{Mb})$ closely matches the length of the gaps in the Pr102 assembly (12.2 Mb).

The flow cytometry analysis of our isolate estimated a genome size of $75 \mathrm{Mb}$; therefore, our overall assembly of $60.5 \mathrm{Mb}$ was substantially less than the experimentally measured value but greater than the estimate of the ND886 genome size based on $k$-mer depth distribution $(52.3 \mathrm{Mb})$ (Supplementary Fig. S11) (Tyler et al. 2006). The reason for the difference between the assembly length and the $k$-mer estimate is not clear, but $k$-mer analysis is not free from artifacts and, in many cases, the values fluctuate (Guo et al. 2015). Following the FALCON assembly, there were 13,300 unmapped reads comprising 133 $\mathrm{Mb}$ of sequences. Using Canu, we could assemble these unmapped reads into nine unplaced contigs $(265 \mathrm{~kb})$. The unplaced contigs were not a part of the associated contigs. Furthermore, there were $23 \mathrm{Mb}$ of unassembled sequences produced by Canu assembler that consisted primarily of short tandem repeats that could not be incorporated into the main assembly. This was largely due to the low coverage and low sequence complexity. This HR component contained a total of 3,275 sequences that were mostly singleton reads. It is not clear whether the low coverage of these sequences is due to poor incorporation into the PacBio sequencing libraries or because these reads contain numerous errors. If the former is the case, then the $P$. ramorum ND886 genome may be as much as $23 \mathrm{Mb}$ longer than we have estimated. Phytophthora species have genomes with HR regions and long stretches of repeats rendering them very difficult to assemble (Haas et al. 2009; Tyler et al. 2006).

The HR component is comparable to the poorly assembled leftovers of other near-complete genomes such as the human genome. In the human genome, unmapped leftover sequences were comprised of pentameric repeats (Fonville et al. 2016). Large numbers of tandem repeat elements were found in the HR component of the ND886 genome. Some of the abundant tandem repeat elements from the HR component are represented in Table 2. These tandem repeat elements from the HR component had no matches to the $P$. sojae V5 assembly. On the other hand, the decameric repeats TCGCCAACGG and AACGGTCGCC were found in both the ND886 and Pr102 V1 assemblies, although in reduced numbers in the Pr102 assembly. This suggests that these tandem repeat elements are either unique to $P$. ramorum or they are missing from the $P$. sojae assembly because they fell into unassembled regions. Telomere repeat elements were searched in the assembled scaffolds and from HR components as described by (Fulnečková et al. 2013), but we could not identify any such elements.

\section{Genome phasing and block analysis.}

Our motivation for phasing the haplotypes of $P$. ramorum was to provide a powerful tool for future population genomics studies on large-scale polymorphisms, understanding points of mitotic recombination, and to better understand the relationship between DNA sequence and RNA expression. For example, it will be valuable to conduct a population level study to determine if a subset of haplotype blocks may be preserved in other asexual lineages of $P$. ramorum or if a number of SNPs are inherited or lost together during large-scale mutation events, such as structural variations or gene conversions. We can also estimate somatic recombination rates to understand its impact on asexual evolution, as somatic recombination can produce diverse allele combinations, which may be followed by gene conversion (Jiang et al. 2013; Lamour et al. 2012; Chamnanpunt et al. 2001). The creation of allelic diversity in an asexually reproducing population such as $P$. ramorum in North America can result in phenotypic diversification and the increase of evolutionary potential. Recently, a sexual population of $P$. ramorum was isolated in Vietnam by T. Jung (Phytophthora Research Centre, Czech Republic) and J. Webber and C. Brasier (U.K. Forest Research). It will be valuable to determine whether any haplotype blocks are shared among the four known asexual P. ramorum lineages (Van Poucke et al. 2012) and with the sexual population in Vietnam.

Haplotype phasing of sequence assemblies can be challenging because it relies on marker density, read length, and read accuracy. By combining CCNV analysis and the ND886 phased genome, we were able to identify potential physical linkages between contigs and their phases, although some uncertainty is introduced by the possibility of mitotic crossovers in individual strains. Without the CCNV analysis, relationships between haplotypes in the different blocks remain unknown, as complete haplotypes could not be inferred. Though the PacBio reads had an average marker density of 0.62 SNPs per $1,000 \mathrm{bp}$ and average read lengths of $10.57 \mathrm{~kb}$, the reads were still not long enough to span all regions with low marker density. In addition, PacBio reads have a high error rate, which makes distinguishing between a heterozygous region and error difficult when coverage is low. For the first 25 largest contigs, SNP density is plotted in Supplementary Fig. S12.

Gene calling was performed on the unpolished primary contigs as well as on the polished haplotypes. Our gene prediction identified 12,337 gene models in the 60.5-Mb ND886 unpolished primary contigs. This number is substantially less than the 16,134 predicted in the Pr102 Sanger assembly. The probable reason for this discrepancy is that the primary contigs produced from Falcon assembly were not polished and had sequencing errors leading to frame-shift errors in coding regions. This subsequently led to genes not called by Augustus and, thus, the number of predicted genes were less than that of the predicted genes of Pr102 V1 (Tyler et al. 2006). From the polished consensus haplotypes, we were able to predict more genes, comparable to Pr102 V1 assembly (14,470 and 14,998 for haplotypes $\mathrm{A}$ and $\mathrm{B}$, respectively). The differences in gene numbers between haplotypes $\mathrm{A}$ and $\mathrm{B}$ result from different numbers of indels being corrected by polishing of the gene models of the two haplotypes. In the unpolished p-contigs, the 345 haplotype blocks overlapped with 11,227 of the 12,337 predicted protein coding genes, which is about $91 \%$ of all the protein coding genes. This includes $328 R X L R$ and $19 C R N$ effector genes.

We checked for the remaining $9 \%$ of protein coding genes outside the haplotype blocks; those genes are enriched in transposon-associated annotations such as reverse transcription, GAG, integrase core domain, DUF 4219, and GAGpreintegrase domain. The annotations also include zinc knuckle, ABC transporter, ankyrin repeats, and sugar efflux transporter for intercellular exchange.

\section{Copy number variation and possible trisomy in Pr102.}

The availability of the phased assembly enabled us to assess possible copy number variation in $P$. ramorum. Comparison of read depth ratios in Pr102 and ND886 revealed that sequences in Pr102 corresponding to several contigs of the phased assembly $(10,43,44,52,66,110$, and 112) do show statistically 
significant CCNV (Table 4). All the relevant regions show a consistent ratio ranging from $1.84: 1.12$ to $1.65: 1: 28$ and averaging 1.75:1.17 in Pr102 compared with 1:1 in ND886 (Table 4). These values were averaged from the output of TitanCNA, which calculates the read depth for each heterozygous locus. Since the sum of the ratios is close to 3.0, it suggests that one or more chromosomes or chromosome regions may be trisomic. Since a clear 2:1 ratio would normally be expected in the case of trisomy, the observed ratio of $1.75: 1.17$ (which was statistically significantly different than $2: 1$ in each region) is surprising. Mitotic recombination or gene conversion seem an unlikely explanation for the nonstoichiometric ratio, as they are predicted to produce a mosaic of genomic segments showing either $2: 1$ or $1: 2$ ratios, rather than the quite consistent ratio of 1.75:1.17. A more plausible explanation is that Pr102 is heterokaryotic, with most nuclei carrying one configuration (e.g., $\mathrm{ABB}$ ) and a minority carrying the complementary configuration (e.g., AAB). However, efforts to isolate genetically purified lines of Pr102 by single zoospore isolation or hyphal tipping have, so far, not been successful. An ABB/AAB heterokaryon might have formed due to chromosome missegregation during mitosis, but it is unusual for heterokaryons to persist as stably as Pr102 has, unless there is strong genetic selection for retention of both nuclear types. However, we have no independent evidence for the presence of such selection, other than the failure to isolate purified lines.

\section{Evolutionary trends within the effectors.}

We identified three kinds of variations in RXLR effector gene sequences in comparisons between alleles and paralogs. One was effector gene pairs in Pr102 and ND886 that shared $>98 \%$ nucleotide sequence similarity. The second was effector gene pairs in Pr102 and ND886 that differed by the presence of one or more indels; in some cases, a frame-shift was introduced, drastically changing the sequence comparisons between the two effectors from that point on, or, if there was no frame-shift, one effector became longer than the other. Given the potential for indels to be introduced during PacBio sequencing, it is difficult to determine how many of these indels are valid. The third category was presence-absence polymorphisms. The missing RXLR genes Avh269, Avh299, Avh9, Avh336, Avh202, and Avh292 appeared to be pseudo-genes that contained frame-shift indels ranging from 2 to 13. Such frameshifts were scattered throughout the genomic regions, including those in which those effector genes are located. Given the deficiencies in the Sanger draft assembly of Pr102, it is difficult to determine how many of these polymorphisms are valid. Wang et al. (2011) observed similar classes of RXLR gene polymorphisms in $P$. sojae. For effector gene comparisons in which indels were absent, we characterized $\mathrm{dN}$ and $\mathrm{dS}$ differences in order to identify genes that might exhibit statistically significant evidence of positive or negative selection. However, no pairs of putative orthologs showed significant evidence of positive or negative selection. Given that both strains are from the NA1 lineage, this may not be surprising.

\section{Bipartite genome architecture in ND886 characterized by location of effector genes in gene-sparse regions.}

In oomycete plant pathogens, RXLR effectors play a very significant role in the host-pathogen interaction. Effectors delivered into the host cell can suppress plant defenses (Qutob et al. 2009). In contrast, those that can be identified by host immune receptors can trigger the plant defense response. This coevolutionary struggle constantly selects for changes in the effector genes. Over time, the genes become unrecognizable through standard gene prediction methods. Therefore, in order to reduce false negatives, refined bioinformatics prediction methods are required to predict the RXLR effectors. We identified 393 and 394 predicted $R X L R$ effector genes in haplotypes $\mathrm{A}$ and $\mathrm{B}$, respectively, of which 24 and 25 (from haplotypes A and B, respectively) were absent from the Pr102 V1 assembly.

CRNs are another class of intracellular effector proteins, many of which target the host nucleus during infection (Stam et al. 2013). We identified 21 and $25 C R N$ genes (in haplotypes $\mathrm{A}$ and $\mathrm{B}$, respectively). Most of these genes were largely present within repeat-rich regions of the ND886 genome that corresponded to gap regions in the Pr102 V1 assembly. In contrast to the $R X L R$ effector genes, the $C R N$ genes never occurred in clusters of more than two members, suggesting a lack of new duplications and an ancient origin of the genes.

A growing number of studies have revealed that filamentous eukaryotic plant pathogens display a bipartite evolutionary genome architecture (Raffaele and Kamoun 2012) and that many effector genes reside in rapidly evolving regions of the genome (Dong et al. 2015). This genome architecture was described for $P$. sojae and $P$. ramorum (Tyler et al. 2006) as well as $P$. infestans (Haas et al. 2009). The locations of $R X L R$ genes may facilitate a process of rapid birth and death evolution, which results in divergent sets of effectors (Win et al. 2007). This, in turn, may enable the pathogen to manipulate the host while evading detection. Here, we show that the primary contig assembly contains numerous repetitive elements $(48.1 \%$ repeats), along with a large set of putative RXLR effectors. Most of the $R X L R$ effector genes were found in the repeat-rich regions of the genome. We also found that $C R N$ genes were located in the gene-sparse, repeat-rich regions. Thus, the haplotype-phased long-range assemblies we have generated strongly confirm the presence of bipartite evolutionary genome architecture in $P$. ramorum.

\section{MATERIALS AND METHODS}

Strain, DNA isolation, and genome sequencing.

$P$. ramorum ND886 (CDFA1418886) was originally isolated from foliage of Camellia cultivar Coral Delight in 2004 in Marin County, California (C. Blomquist, California Department of Food and Agriculture, personal communication), and is the standard isolate used at the National Ornamental Research site at the Dominican University of California. For production of $P$. ramorum mycelia for extraction of highmolecular weight DNA, a small mycelial plug was transferred to each petri plate $(6 \mathrm{~cm}$ diameter) containing $7 \mathrm{ml}$ of Ribeiro's synthetic medium (Ribeiro et al. 1975) with $1.5 \%$ agar overlaid with a polycarbonate membrane filter (VWR) and was then grown for 8 days at $21^{\circ} \mathrm{C}$ in the dark. Each circular mycelial mat was then lifted from the polycarbonate membrane surface, was transferred to a 2-ml microcentrifuge tube, and was immediately snap-frozen in liquid nitrogen. Each sample weighed approximately $150 \mathrm{mg}$. A total of about $6 \mathrm{~g}$ of tissue was pulverized, using mortar and pestle, in liquid nitrogen. Subsequently, we followed a nuclei isolation method (Zhang et al. 2012) to obtain DNA fragments larger than $100 \mathrm{~kb}$. In essence, the ground tissue was homogenized in nuclear isolation buffer (NIB), was filtered through cheesecloth and Miracloth, and was then pelleted by centrifugation. After a total of three washes with the ice-cold NIB buffer, the nuclei pellet was resuspended in $10 \mathrm{ml}$ of buffer G2 (general lysis buffer) and DNA was extracted, using the Qiagen Genomic-tip 100/G procedure (Pacific Biosciences 2013). A 40- $\mu$ g high-molecular weight DNA sample was sent to the University of California Davis Genome Center, where the PacBio SMRT library was constructed and sequenced on the PacBio RS II SMRT instrument with P6-C4 chemistry. A total of 402,170 reads with an average 
length of $10.5 \mathrm{~kb}$ were generated, producing $70 \times$ coverage of the $60.5-\mathrm{Mb}$ genome.

\section{Illumina sequencing for isolates ND886 and Pr102.}

Three Illumina paired-end libraries were generated from ND886 genomic DNA in 2016 and 2017. The first library, generated in June 2016, had 44 million reads, a 350 bp insert size, and $100 \mathrm{bp}$ read length, accounting for $55 \times$ coverage. The second library was generated in July 2017 with 46 million reads, a 350 bp average insert size, and $100 \mathrm{bp}$ average read length, accounting for $63 \times$ coverage. The third library was generated in August 2017, had 41 reads, a 350 bp insert size, and $150 \mathrm{bp}$ average read length, accounting for $86 \times$ coverage. Illumina paired-end libraries were also generated for Pr102. These libraries were sequenced in November 2012 and had 70 million reads, with a $350 \mathrm{bp}$ insert size and $100 \mathrm{bp}$ read lengths. The read coverage was $67 \times$. Read depth was calculated using SAMtools (Li et al. 2009).

\section{Genome assembly of $P$. ramorum ND886.}

The $P$. ramorum ND886 genome was assembled with the FALCON assembler. PacBio long reads were directly provided to the assembler. The FALCON assembler was used to generate the draft primary contig assembly $(60.2 \mathrm{Mb}$ p-contigs) and associated contigs (18 Mb a-contigs). The description of primary and associated contigs is provided above. We mapped the reads from the three Illumina libraries as well as the PacBio reads to the primary contigs for haplotype phasing and allele comparisons. The Illumina reads were used for polishing the consensus haplotypes, using Pilon (Walker et al. 2014).

\section{Unplaced contigs and HR component from unmapped reads.}

The primary assembly and the associated contigs generated using the FALCON assembler (discussed above) were aligned with the PacBio raw reads using BLASR (Chaisson and Tesler 2012). A total of 13,300 PacBio reads comprising approximately $134 \mathrm{Mb}$ of sequences were found to be unmapped with the assembly. The unmapped reads were provided to the Canu assembler, resulting in nine contigs totaling $265 \mathrm{~kb}$, which we named unplaced contigs, plus a collection of unassembled low-coverage sequences totaling $23 \mathrm{Mb}$, named the $\mathrm{HR}$ component.

\section{Haplotype phasing and comparisons of alleles from phased haplotypes.}

The Illumina reads were mapped to the reference genome assembly produced by FALCON (60.2 Mb of p-contig) using BWA-mem (Li 2013). SAMtools (Li et al. 2009) and BCFtools (Li 2011) were used to call the genotypes to generate the variant information. We filtered the genotype data, for only heterozygous sites, using SnpEff (Cingolani et al. 2012) and compared all heterozygous loci in all three Illumina libraries of ND886 that were sequenced in Jun 2016, July 2017, and August 2017. After mapping Illumina reads to the PacBio assembled reference genome, we noticed that a subset of heterozygous sites included loci that had two alternative alleles representing the genotype (e.g., 1/2). We reasoned that these sites were sequencing errors in the Illumina libraries because the PacBio reference allele was not represented. The PacBio raw reads were mapped with the reference assembly using BLASR (Chaisson and Tesler 2012). The alignment produced from PacBio reads as well as the genotype data from the three Illumina libraries were used for phasing, using WhatsHap (Garg et al. 2016). The assembled haplotigs were arbitrarily categorized as haplotypes A and B within each block. The consensus was called on the haplotypes individually. The individual
PacBio reads are known to have major high indel error rates. Therefore, to reduce the indel errors in our assembly, after phasing the heterozygous SNP sites, we used the Illumina reads to do consensus calling on the haplotypes (polishing) to reduce the indel errors from the assembly, using Pilon.

The comparisons of allele-specific genes in the newly predicted $R X L R$ genes from the consensus haplotypes were compared with the reference genome assembly of primary contigs.

\section{CCNVs and haplotype blocks.}

To characterize the relationships between haplotype blocks, we studied copy number variations that are potentially physically linked and that occurred in Pr102. The read-depth method of TitanCNA (Ha et al. 2014) was implemented, using the ND886 haplotype-phased genome assembly as reference. The copy number variations presented in this paper span whole contigs or large fractions of contigs that suggest at least partial aneuploidy and, therefore, we have used the term CCNV. TitanCNA was originally developed to study copy number variation in human cancer, which reproduces clonally, and the program was designed for paired tumor-normal studies. Like cancer, $P$. ramorum NA1 lineage reproduces clonally. A population analysis estimates that there are only about $0.004 \%$ SNP difference (716 SNV versus 17,056,937 heterozygous SNPs), which further supports the assumption of clonality. The ND886 reference was used as a control to detect somatic variations in the form of CCNVs. With ND886 as a control, we were able to reduce false positives that arise from technical errors. For example, certain regions of the genome have read biases that lead to uneven coverage. Simply calculating the read depth of Pr102 directly could mistakenly identify one of these biased regions as a CCNV. The TitanCNA workflow was modified to accommodate a phased genome. First, TitanCNA requires heterozygous SNP loci from a control sample. To obtain heterozygous sites of ND886, adapter trimmed reads of the control were mapped, using bwa aligner, to both FASTA files that represent haplotype A and B. The two BAM files from each haplotype were merged into a single BAM file. Genotypes were called using SAMtools mpileup and Bcftools and were then filtered for heterozygous loci with SnpSift (Cingolani et al. 2012). Second, TitanCNA requires loci and allele read depth information from both the variant and control isolate. The same adapter-trimmed reads of step 1 were binned based on best match, using BBSplit (GitHub) to both haplotype FASTA files. Reads were sorted based on a 0.56 alignment score ratio between references. Reads were binned to the best matching haplotype to avoid aligning reads to all haplotypes at once, which would lower the MAPQ score (reads would have twice the number of matching regions). Most of the 100-bp Illumina reads $(86.2 \%)$ were ambiguous because they did not span heterozygous SNPs; these reads were randomly assigned between either haplotype references. Ambiguous reads were included because removing the ambiguous reads resulted in similar CCNV results but with fewer datapoints because the read depth decreased. Reads were then aligned to the haplotype they best matched using bwa aligner. For each isolate, BAM files of both haplotypes were merged. WIG (read depth) files were generated from the BAM files for the variant and control samples using HMMcopy (Lai and Shah 2012). After filtering for a mapping quality score of 20 or greater, TitanCNA extracted read depth information of each SNP to predict regions of $\mathrm{CNV}$.

To test the statistical significance of contig-wide CCNV signals, a Welch's $t$ test on ranked data was calculated for the log ratios of each such contig from each haplotype (e.g., contig 10 from haplotype A) compared with a control contig with a $1: 1$ ratio (contig $9 ; 0.03$ mean $\log$ ratio) and to a hypothetical contig 
with a 2:1 read depth (contig nine of haplotype B in which 1.0 was added to each individual $\log$ ratio to produce a median $\log$ ratio of 1.02 and a mean $\log$ ratio of 1.03 ).

\section{Assessments of genome assemblies of isolates.}

The $P$. ramorum $\mathrm{V} 1$ assembly and the $62.5-\mathrm{Mb}$ ND886 FALCON assembly were assessed by using Quast (Gurevich et al. 2013) for $\mathrm{N}_{50}$ values and for the evaluation of gaps. The core genes were assessed using BUSCO 2.0 (Simão et al. 2015), with COG categories from Stramenopiles. We also estimated the genome size by $k$-mer analysis using jellyfish 2.2.7 (Marçais and Kingsford 2011), using 25 as the $k$-mer value. There were two peaks at 70 and 140 coverage corresponding to single haplotypes and both haplotypes, respectively. The genome size was computed as the sum of the frequencies of all 25-mers in the Illumina reads (excluding those with low coverage <17) divided by peak coverage value for both haplotypes (140). This indicated a possible genome size of $52.3 \mathrm{Mb}$.

\section{Flow cytometry.}

Mycelia of P. Ramorum isolates ND886 and Pr102 were taken from V8 plates (20\% V8 Juice with $1.5 \%$ agar) and grown in test tubes containing $5 \mathrm{ml}$ filter-sterilized $5 \%$ clarified V8 broth (Englander and Roth 1980) for 7 days at $21^{\circ} \mathrm{C}$. The mycelium was harvested and washed three times with sterile water. Arabidopsis thaliana Col-0 was chosen as the internal DNA reference standard with a genome size of $1 \mathrm{C}=$ $157 \mathrm{Mb}$ (Doležel and Bartoš 2005). A modified protocol derived from (Bertier et al. 2013) was then followed. In brief, nuclei extraction was done using the Cystain PI absolute P kit (Sysmex America Inc.). For each sample approximately five flower buds of $A$. thaliana and a small amount of $P$. ramorum mycelium (around $1 \mathrm{mg}$ of dry blotted mycelium) were combined and chopped with a razor blade (Gillette) in a Petri dish containing $500 \mu \mathrm{l}$ of extraction buffer. After chopping, the suspension was filtered through a $10-\mu \mathrm{m}$ filter (CellTrics, Sysmex America Inc.) and $2 \mathrm{ml}$ of a propidium iodide staining solution was added. The samples were incubated for $60 \mathrm{~min}$ in the dark at $4^{\circ} \mathrm{C}$. Measurements were done on a Becton Dickinson FACScan (Franklin Lakes, NewJersey) equipped with a $488 \mathrm{~nm}$ laser and a $585 / 42 \mathrm{~nm}$ band pass filter. The data were analyzed using FlowJo v.10, and DNA content was subsequently inferred, using a linear regression with the ratios between peak positions of the $P$. ramorum sample and the Arabidopsis size standard.

\section{Gene prediction and genome annotation.}

The gene prediction was performed on the genome assembly (primary contig), unplaced contigs, and haplotype phased consensus assembly (A and $\mathrm{B})$, using $P$. ramorum $\mathrm{V} 1$ gene models as an initial training dataset, using Scipio (Keller et al. 2008). Then the initial round of gene predictions was used to retrain Augustus (Stanke and Waack 2003) before repeating the gene prediction. The unassembled low-coverage sequences were mined for the tandem repeat elements using Tandem Repeat Finder (Benson 1999).

The proteins predicted from the phased consensus haplotypes (A and B) as well as P. sojae V5 were searched in the dbCAN database to find the CAZ carbohydrate-active enzymesassociated proteins. To identify the CAZy families that are predicted to be associated with pathogen-host interactions, the Pathogen-Host Interaction database (Winnenburg et al. 2006) was searched, by BLASTp, using the CAZy gene families of $P$. ramorum ND886 haplotypes (A and $\mathrm{B}$ ) and $P$. sojae as queries. In addition, the Pfam annotation was done for the primary reference assembly, using interproscan (Jones et al. 2014).

\section{$R X L R$ and $C R N$ gene family prediction.}

Initially, we tried predicting the $R X L R$ genes from already available methods from the Galaxy pipeline (Giardine et al. 2005), using the models of Win et al. (2007) and Whisson et al. (2007). By this method, we were able to predict 310 and 151 $R X L R$ genes from haplotype $\mathrm{A}$, using the two models, respectively, and 307 and 151 from haplotype B. Ultimately, however, we used a modified version of the statistically based method of Jiang et al. (2008) for predicting effectors. The EMBOSS Getorf program was used for extracting all open reading frames (ORFs) with a minimum nucleotide length of $300 \mathrm{bp}$ from the haplotype-phased consensus assemblies. As an initial step, the $P$. ramorum Pr102 V1 Avh HMM models (Jiang et. al. 2008) were used for searching for Avh protein sequences from the extracted ORFs. The HMM score cutoff E-value $<1 \mathrm{e}-5$ was chosen for further downstream data analysis. The candidates that passed the criteria were used for signal peptide identification, using SignalP 3.0. (Bendtsen et al. 2004). Those with predicted signal peptides were screened by TMHMM v2.0 (Krogh et al. 2001) to remove any proteins with predicted transmembrane domains. Proteins lacking any organellar localization, as predicted by TargetP (Emanuelsson et al. 2007), were further selected for glycosylphosphatidylinositol (GPI) anchor prediction using PredGPI (Pierleoni et al. 2008). Sequences lacking any GPI signals were subsequently used for motif prediction using meme (Bailey et al. 2009), searching for the motifs RXLR, DEER, and W-Y-L (Jiang et al. 2008). We also used multiple rounds of Blast searches using the Pr102 V1 effectors as queries against the consensus haplotypes, as a complementary means to identify RXLR effectors. We took the union of the effector collections obtained using HMM and blast methods and retained the longest effectors lacking an internal stop codon as the final copy. We transferred the identities of previously predicted effector genes if the sequences matched $>98 \%$ nucleotide identity (with a few exceptions). We named new effector genes if they had $<90 \%$ identity with any of the existing previously predicted effectors.

For the $C R N$ genes, the HMM models were built from published CRN effectors as described by Yin et al. (2015) and used to query the phased assembly. We searched for effectors in the unplaced contigs but did not find any.

\section{Transposon prediction.}

Transposons from phased consensus haplotypes and Pr102 V1 assembly were predicted using TransposonPSI (Haas 2007).

\section{Renaming of several effectors from the Pr102 V1 assembly.}

In this study, we used a nomenclature system in which, if two genes encode a nearly identical effector amino acid sequence (1 to 3 amino acid differences), suffixes, such as PrAvh1a and PrAvh1b, were used. If two genes encoded exactly identical proteins, then a combination of character and numerical suffixes were used, e.g., PrAvh1a1, PrAvh1a2. For completely new effector genes (having less than $90 \%$ identity), new numbering was used. Based on this, we renamed effectors such as PrAvh138, PrAvh190, PrAvh213, PrAvh221, PrAvh274, PrAvh275, PrAvh340, PrAvh386, PrAvh92 and PrAvh91. For example, PrAvh138 and PrAvh139 have only two amino acid differences so are paralogs as per our nomenclature. So, e.g., PrAvh138 was renamed PrAvh138a and PrAvh139 was named PrAvh138b.

\section{Genome architecture analysis}

for the $P$. ramorum ND886 phased consensus haplotypes.

The genome architecture was analyzed by calculating the flanking intergenic regions of every gene in the consensus 
haplotype-phased genome. To determine the architecture of the genome, the distances of each gene (including effector genes) to its closest gene neighbors in the $5^{\prime}$ and $3^{\prime}$ directions were calculated from the gff co-ordinate file, using $\mathrm{R}$ and Perl scripts. Two-dimensional data binning was then performed (Saunders et al. 2014).

\section{dN/dS analysis.}

For assessing selection pressures acting upon RXLR effectors, the effector genes from the consensus haplotypes were compared with those from Pr102. We identified orthologs using the MCL clustering algorithm (Dongen 2000) from OrthoMCL (Li et al. 2003). Gene sequences from haplotype A and B were clustered into 120 ortholog groups. For each $R X L R$ gene pair within each ortholog group, the Yn00 program was used to calculate the $\mathrm{dN}$ and $\mathrm{dS}$ values and their standard errors. To mitigate loss of power due to multiple testing, each ND886 $R X L R$ effector gene was paired only with its closest homolog in Pr102 (putatively its ortholog). Gene pairs differing by indels were not included in the analysis. Using these data, the significance of the difference between each $\mathrm{dN}$ and $\mathrm{dS}$ score was calculated as a two-tailed $P$ value, which was then adjusted for multiple testing, using the method of Benjamini and Hochberg (1995). Differences between $\mathrm{dN}$ and dS with an adjusted $P$ value $<0.025$ were judged significant. Genes for which $\mathrm{dN}$ was significantly greater than dS were predicted to be subject to positive, diversifying selection. Those for which dS was significantly greater than $\mathrm{dN}$ were predicted to be subject to negative, purifying selection.

\section{ACKNOWLEDGMENTS}

The authors acknowledge Computational Genomics Lab members for valuable suggestions, P. Das for improving the resolution of figures, D. Sen from Computational Genomics Lab, CSIR-IICB for valuable discussion, W. Ye for providing previous HMM models for Avh data. We thank M. Martin and T. Marschall, the whatsHap developers for suggestions on haplotype phasing, L. Bertier for flow cytometry analysis, C. Blomquist and D. Rizzo for providing the isolates ND886 and Pr102, respectively, and associated information.

\section{LITERATURE CITED}

Bailey, T. L., Boden, M., Buske, F. A., Frith, M., Grant, C. E., Clementi, L., Ren, J., Li, W. W., and Noble, W. S. 2009. MEME SUITE: Tools for motif discovery and searching. Nucleic Acids Res. 37:W202-W208.

Beattie, D. 2009. The potential for hybridisation between resident and invasive Phytophthora pathogens on trees. Imperial College, London.

Bendtsen, J. D., Nielsen, H., von Heijne, G., and Brunak, S. 2004. Improved prediction of signal peptides: SignalP 3.0. J. Mol. Biol. 340:783-795.

Benjamini, Y., and Hochberg, Y. 1995. Controlling the false discovery rate: A practical and powerful approach to multiple testing. J. R. Stat. Soc. B 57:289-300.

Benson, G. 1999. Tandem repeats finder: A program to analyze DNA sequences. Nucleic Acids Res. 27:573-580.

Bertier, L., Leus, L., D'hondt, L., de Cock, A. W., and Höfte, M. 2013. Host adaptation and speciation through hybridization and polyploidy in Phytophthora. PLoS One 8:e85385.

Brouwer, H., Coutinho, P. M., Henrissat, B., and de Vries, R. P. 2014. Carbohydrate-related enzymes of important Phytophthora plant pathogens. Fungal Genet. Biol. 72:192-200.

Chaisson, M. J., and Tesler, G. 2012. Mapping single molecule sequencing reads using basic local alignment with successive refinement (BLASR): Application and theory. BMC Bioinformatics 13:238.

Chamnanpunt, J., Shan, W.-X., and Tyler, B. M. 2001. High frequency mitotic gene conversion in genetic hybrids of the oomycete Phytophthora sojae. Proc. Natl. Acad. Sci. U.S.A. 98:14530-14535.

Chin, C. S., Peluso, P., Sedlazeck, F. J., Nattestad, M., Concepcion, G. T., Clum, A., Dunn, C., O’Malley, R., Figueroa-Balderas, R., Morales-Cruz, A., Cramer, G. R., Delledonne, M., Luo, C., Ecker, J. R., Cantu, D., Rank, D. R., and Schatz, M. C. 2016. Phased diploid genome assembly with single-molecule real-time sequencing. Nat. Methods 13:1050-1054.
Cingolani, P., Platts, A., Wang, L., Coon, M., Nguyen, T., Wang, L., Land, S. J., Lu, X., and Ruden, D. M. 2012. A program for annotating and predicting the effects of single nucleotide polymorphisms, SnpEff: SNPs in the genome of Drosophila melanogaster strain w1118; iso-2; iso-3. Fly (Austin) 6:80-92.

Doležel, J., and Bartoš, J. 2005. Plant DNA flow cytometry and estimation of nuclear genome size. Ann. Bot. 95:99-110.

Dong, S., Raffaele, S., and Kamoun, S. 2015. The two-speed genomes of filamentous pathogens: Waltz with plants. Curr. Opin. Genet. Dev. 35: 57-65.

Dongen, S, 2000. Performance criteria for graph clustering and Markov cluster experiments. Ph.D. thesis. University of Utrecht, The Netherlands. Published online. doi:10.1.1.26.9783

Elliott, M., Yuzon, J., C, M. M., Tripathy, S., Bui, M., Chastagner, G. A., Coats, K., Rizzo, D. M., Garbelotto, M., and Kasuga, T. 2018. Characterization of phenotypic variation and genome aberrations observed among Phytophthora ramorum isolates from diverse hosts. BMC Genomics 19:320.

Emanuelsson, O., Brunak, S., von Heijne, G., and Nielsen, H. 2007. Locating proteins in the cell using TargetP, SignalP and related tools. Nat. Protoc. 2:953-971.

Englander, L., and Roth, L. 1980. Interaction of light and sterol on sporangium and chlamydospore production by Phytophthora lateralis. Phytopathology 70:650-654.

English, A. C., Richards, S., Han, Y., Wang, M., Vee, V., Qu, J., Qin, X., Muzny, D. M., Reid, J. G., Worley, K. C., and Gibbs, R. A. 2012. Mind the gap: Upgrading genomes with Pacific Biosciences RS long-read sequencing technology. PLoS One 7:e47768.

Fonville, N. C., Velmurugan, K. R., Tae, H., Vaksman, Z., McIver, L. J., and Garner, H. R. 2016. Genomic leftovers: Identifying novel microsatellites, over-represented motifs and functional elements in the human genome. Sci. Rep. 6:27722.

Fulnečková, J., Sevcíková, T., Fajkus, J., Lukesová, A., Lukeš, M., Vlček, C., Lang, B. F., Kim, E., Eliás, M., and Sykorová, E. 2013. A broad phylogenetic survey unveils the diversity and evolution of telomeres in eukaryotes. Genome Biol. Evol. 5:468-483.

Garg, S., Martin, M., and Marschall, T. 2016. Read-based phasing of related individuals. Bioinformatics 32:i234-i242.

Giardine, B., Riemer, C., Hardison, R. C., Burhans, R., Elnitski, L., Shah, P., Zhang, Y., Blankenberg, D., Albert, I., Taylor, J., Miller, W., Kent, W. J., and Nekrutenko, A. 2005. Galaxy: A platform for interactive largescale genome analysis. Genome Res. 15:1451-1455.

Gnerre, S., MacCallum, I., Przybylski, D., Ribeiro, F. J., Burton, J. N., Walker, B. J., Sharpe, T., Hall, G., Shea, T. P., Sykes, S., Berlin, A. M., Aird, D., Costello, M., Daza, R., Williams, L., Nicol, R., Gnirke, A., Nusbaum, C., Lander, E. S., and Jaffe, D. B. 2011. High-quality draft assemblies of mammalian genomes from massively parallel sequence data. Proc. Natl. Acad. Sci. U.S.A. 108:1513-1518.

Goss, E. M., Press, C. M., and Grünwald, N. J. 2013. Evolution of RXLRclass effectors in the oomycete plant pathogen Phytophthora ramorum. PLoS One 8:e79347.

Grünwald, N. J., Goss, E. M., and Press, C. M. 2008. Phytophthora ramorum: A pathogen with a remarkably wide host range causing sudden oak death on oaks and ramorum blight on woody ornamentals. Mol. Plant Pathol. 9:729-740.

Guo, L. T., Wang, S. L., Wu, Q. J., Zhou, X. G., Xie, W., and Zhang, Y. J. 2015 . Flow cytometry and $k$-mer analysis estimates of the genome sizes of Bemisia tabaci B and Q (Hemiptera: Aleyrodidae). Front. Physiol. 6: 144.

Gurevich, A., Saveliev, V., Vyahhi, N., and Tesler, G. 2013. QUAST: Quality assessment tool for genome assemblies. Bioinformatics 29: 1072-1075.

Ha, G., Roth, A., Khattra, J., Ho, J., Yap, D., Prentice, L. M., Melnyk, N., McPherson, A., Bashashati, A., Laks, E., Biele, J., Ding, J., Le, A., Rosner, J., Shumansky, K., Marra, M. A., Gilks, C. B., Huntsman, D. G., McAlpine, J. N., Aparicio, S., and Shah, S. P. 2014. TITAN: Inference of copy number architectures in clonal cell populations from tumor wholegenome sequence data. Genome Res. 24:1881-1893.

Haas B, 2007. TransposonPSI: An application of PSI-blast to mine (Retro-) transposon ORF homologies. Broad Institute, Cambridge, MA, U.S.A.

Haas, B. J., Kamoun, S., Zody, M. C., Jiang, R. H., Handsaker, R. E., Cano, L. M., Grabherr, M., Kodira, C. D., Raffaele, S., Torto-Alalibo, T., Bozkurt, T. O., Ah-Fong, A. M., Alvarado, L., Anderson, V. L., Armstrong, M. R., Avrova, A., Baxter, L., Beynon, J., Boevink, P. C., Bollmann, S. R., Bos, J. I., Bulone, V., Cai, G., Cakir, C., Carrington, J. C., Chawner, M., Conti, L., Costanzo, S., Ewan, R., Fahlgren, N., Fischbach, M. A., Fugelstad, J., Gilroy, E. M., Gnerre, S., Green, P. J., Grenville-Briggs, L. J., Griffith, J., Grünwald, N. J., Horn, K., Horner, 
N. R., Hu, C. H., Huitema, E., Jeong, D. H., Jones, A. M., Jones, J. D., Jones, R. W., Karlsson, E. K., Kunjeti, S. G., Lamour, K., Liu, Z., Ma, L., Maclean, D., Chibucos, M. C., McDonald, H., McWalters, J., Meijer, H. J., Morgan, W., Morris, P. F., Munro, C. A., O’Neill, K., OspinaGiraldo, M., Pinzón, A., Pritchard, L., Ramsahoye, B., Ren, Q., Restrepo, S., Roy, S., Sadanandom, A., Savidor, A., Schornack, S., Schwartz, D. C., Schumann, U. D., Schwessinger, B., Seyer, L., Sharpe, T., Silvar, C., Song, J., Studholme, D. J., Sykes, S., Thines, M., van de Vondervoort, P. J., Phuntumart, V., Wawra, S., Weide, R., Win, J., Young, C., Zhou, S., Fry, W., Meyers, B. C., van West, P., Ristaino, J., Govers, F., Birch, P. R., Whisson, S. C., Judelson, H. S., and Nusbaum, C. 2009. Genome sequence and analysis of the Irish potato famine pathogen Phytophthora infestans. Nature 461:393-398.

Jiang, R. H., de Bruijn, I., Haas, B. J., Belmonte, R., Löbach, L., Christie, J., van den Ackerveken, G., Bottin, A., Bulone, V., Díaz-Moreno, S. M., Dumas, B., Fan, L., Gaulin, E., Govers, F., Grenville-Briggs, L. J., Horner, N. R., Levin, J. Z., Mammella, M., Meijer, H. J., Morris, P., Nusbaum, C., Oome, S., Phillips, A. J., van Rooyen, D., Rzeszutek, E., Saraiva, M., Secombes, C. J., Seidl, M. F., Snel, B., Stassen, J. H., Sykes, S., Tripathy, S., van den Berg, H., Vega-Arreguin, J. C., Wawra, S., Young, S. K., Zeng, Q., Dieguez-Uribeondo, J., Russ, C., Tyler, B. M., and van West, P. 2013. Distinctive expansion of potential virulence genes in the genome of the oomycete fish pathogen Saprolegnia parasitica. PLoS Genet. 9:e1003272.

Jiang, R. H., Tripathy, S., Govers, F., and Tyler, B. M. 2008. RXLR effector reservoir in two Phytophthora species is dominated by a single rapidly evolving superfamily with more than 700 members. Proc. Natl. Acad. Sci. U.S.A. 105:4874-4879.

Jiang, R. H., and Tyler, B. M. 2012. Mechanisms and evolution of virulence in oomycetes. Annu. Rev. Phytopathol. 50:295-318.

Jones, P., Binns, D., Chang, H.-Y., Fraser, M., Li, W., McAnulla, C., McWilliam, H., Maslen, J., Mitchell, A., Nuka, G., Pesseat, S., Quinn, A. F., Sangrador-Vegas, A., Scheremetjew, M., Yong, S. Y., Lopez, R., and Hunter, S. 2014. InterProScan 5: Genome-scale protein function classification. Bioinformatics 30:1236-1240.

Käll, L., Krogh, A., and Sonnhammer, E. L. 2004. A combined transmembrane topology and signal peptide prediction method. J. Mol. Biol. 338:1027-1036.

Kasuga, T., Bui, M., Bernhardt, E., Swiecki, T., Aram, K., Cano, L. M., Webber, J., Brasier, C., Press, C., Grünwald, N. J., Rizzo, D. M., and Garbelotto, M. 2016. Host-induced aneuploidy and phenotypic diversification in the sudden oak death pathogen Phytophthora ramorum. BMC Genomics 17:385.

Kasuga, T., Kozanitas, M., Bui, M., Hüberli, D., Rizzo, D. M., and Garbelotto, M. 2012. Phenotypic diversification is associated with hostinduced transposon derepression in the sudden oak death pathogen Phytophthora ramorum. PLoS One 7:e34728.

Keller, O., Odronitz, F., Stanke, M., Kollmar, M., and Waack, S. 2008. Scipio: Using protein sequences to determine the precise exon/intron structures of genes and their orthologs in closely related species. BMC Bioinformatics 9:278.

Koren, S., Walenz, B. P., Berlin, K., Miller, J. R., Bergman, N. H., and Phillippy, A. M. 2017. Canu: Scalable and accurate long-read assembly via adaptive $k$-mer weighting and repeat separation. Genome Res. 27:722-736.

Krogh, A., Larsson, B., von Heijne, G., and Sonnhammer, E. L. 2001. Predicting transmembrane protein topology with a hidden Markov model: Application to complete genomes. J. Mol. Biol. 305:567-580.

Lai D, Shah S, 2012. HMMcopy: Copy number prediction with correction for GC and mappability bias for HTS data. R package, version $1 . \mathrm{R}$ Project for Statistical Computing.

Lam, H. Y. K., Clark, M. J., Chen, R., Chen, R., Natsoulis, G., O’Huallachain, M., Dewey, F. E., Habegger, L., Ashley, E. A., Gerstein, M. B., Butte, A. J., Ji, H. P., and Snyder, M. 2012. Performance comparison of whole-genome sequencing platforms. Nature Biotechnol. 30:78-82.

Lamour, K. H., Mudge, J., Gobena, D., Hurtado-Gonzales, O. P., Schmutz, J., Kuo, A., Miller, N. A., Rice, B. J., Raffaele, S., Cano, L. M., Bharti, A. K., Donahoo, R. S., Finley, S., Huitema, E., Hulvey, J., Platt, D., Salamov, A., Savidor, A., Sharma, R., Stam, R., Storey, D., Thines, M., Win, J., Haas, B. J., Dinwiddie, D. L., Jenkins, J., Knight, J. R., Affourtit, J. P., Han, C. S., Chertkov, O., Lindquist, E. A., Detter, C., Grigoriev, I. V., Kamoun, S., and Kingsmore, S. F. 2012. Genome sequencing and mapping reveal loss of heterozygosity as a mechanism for rapid adaptation in the vegetable pathogen Phytophthora capsici. Mol. PlantMicrobe Interact 25:1350-1360.

Li, H. 2011. A statistical framework for SNP calling, mutation discovery, association mapping and population genetical parameter estimation from sequencing data. Bioinformatics 27:2987-2993.
Li, H. 2013. Aligning sequence reads, clone sequences and assembly contigs with BWA-MEM. Cornell University, Ithaca, NY, U.S.A. Published online. doi: arXiv:1303.3997

Li, H., and Durbin, R. 2009. Fast and accurate short read alignment with Burrows-Wheeler transform. Bioinformatics 25:1754-1760.

Li, H., Handsaker, B., Wysoker, A., Fennell, T., Ruan, J., Homer, N., Marth, G., Abecasis, G., and Durbin, R.; 1000 Genome Project Data Processing Subgroup. 2009. The sequence alignment/map format and SAMtools. Bioinformatics 25:2078-2079.

Li, L., Stoeckert, C. J., Jr., and Roos, D. S. 2003. OrthoMCL: Identification of ortholog groups for eukaryotic genomes. Genome Res. 13:2178-2189.

Marçais, G., and Kingsford, C. 2011. A fast, lock-free approach for efficient parallel counting of occurrences of $k$-mers. Bioinformatics 27: 764-770.

Pacific Biosciences 2013. Preparing Arabidopsis genomic DNA for sizeselected $\sim 20 \mathrm{~kb}$ SMRTbell ${ }^{\mathrm{TM}}$ Libraries. PacBio SampleNet, Menlo Park, CA, U.S.A. Published online.

Panda, A., Sen, D., Ghosh, A., Gupta, A., C, M. M., Prakash Mishra, G., Singh, D., Ye, W., Tyler, B. M., and Tripathy, S. 2018. EumicrobeDBLite: A lightweight genomic resource and analytic platform for draft oomycete genomes. Mol. Plant Pathol. 19:227-237.

Pierleoni, A., Martelli, P. L., and Casadio, R. 2008. PredGPI: A GPI-anchor predictor. BMC Bioinformatics 9:392.

Qutob, D., Tedman-Jones, J., Dong, S., Kuflu, K., Pham, H., Wang, Y., Dou, D., Kale, S. D., Arredondo, F. D., Tyler, B. M., and Gijzen, M. 2009 Copy number variation and transcriptional polymorphisms of Phytophthora sojae RXLR effector genes Avr1a and Avr3a. PLoS One 4:e5066.

Raffaele, S., Farrer, R. A., Cano, L. M., Studholme, D. J., MacLean, D., Thines, M., Jiang, R. H., Zody, M. C., Kunjeti, S. G., Donofrio, N. M., Meyers, B. C., Nusbaum, C., and Kamoun, S. 2010. Genome evolution following host jumps in the Irish potato famine pathogen lineage. Science 330:1540-1543.

Raffaele, S., and Kamoun, S. 2012. Genome evolution in filamentous plant pathogens: Why bigger can be better. Nat. Rev. Microbiol. 10:417-430.

Rhoads, A., and Au, K. F. 2015. PacBio Sequencing and Its Applications. Genomics Proteomics Bioinformatics 13:278-289.

Ribeiro, O. K., Erwin, D. C., and Zentmyer, G. A. 1975. An improved synthetic medium for oospore production and germination of several Phytophthora species. Mycologia 67:1012-1019.

Saunders, D. G., Win, J., Kamoun, S., and Raffaele, S. 2014. Twodimensional data binning for the analysis of genome architecture in filamentous plant pathogens and other eukaryotes. Pages 29-51 in: PlantPathogen Interactions. Methods in Molecular Biology, Vol. 1127. P. Birch, J. Jones, and J. Bos, eds. Humana Press, Totowa, NJ, U.S.A.

Schornack, S., van Damme, M., Bozkurt, T. O., Cano, L. M., Smoker, M., Thines, M., Gaulin, E., Kamoun, S., and Huitema, E. 2010. Ancient class of translocated oomycete effectors targets the host nucleus. Proc. Natl. Acad. Sci. U.S.A. 107:17421-17426.

Seo, J.-S., Rhie, A., Kim, J., Lee, S., Sohn, M. H., Kim, C. U., Hastie, A., Cao, H., Yun, J. Y., Kim, J., Kuk, J., Park, G. H., Kim, J., Ryu, H., Kim, J., Roh, M., Baek, J., Hunkapiller, M. W., Korlach, J., Shin, J. Y., and Kim, C. 2016. De novo assembly and phasing of a Korean human genome. Nature 538:243-247.

Simão, F. A., Waterhouse, R. M., Ioannidis, P., Kriventseva, E. V., and Zdobnov, E. M. 2015. BUSCO: Assessing genome assembly and annotation completeness with single-copy orthologs. Bioinformatics 31:3210-3212.

Smit, A., Hubley, R., and Green, P. 2016. RepeatMasker Open-4.0. 2015. Institute for Systems Biology, Seattle, WA, U.S.A.

Stam, R., Jupe, J., Howden, A. J., Morris, J. A., Boevink, P. C., Hedley, P. E., and Huitema, E. 2013. Identification and characterisation of CRN effectors in Phytophthora capsici show modularity and functional diversity. PLoS One 8:e59517.

Stanke, M., and Waack, S. 2003. Gene prediction with a hidden Markov model and a new intron submodel. Bioinformatics 19 (Suppl 2): ii215-ii225.

Tyler, B. M., Tripathy, S., Zhang, X., Dehal, P., Jiang, R. H., Aerts, A., Arredondo, F. D., Baxter, L., Bensasson, D., Beynon, J. L., Chapman, J., Damasceno, C. M., Dorrance, A. E., Dou, D., Dickerman, A. W., Dubchak, I. L., Garbelotto, M., Gijzen, M., Gordon, S. G., Govers, F., Grunwald, N. J., Huang, W., Ivors, K. L., Jones, R. W., Kamoun, S., Krampis, K., Lamour, K. H., Lee, M. K., McDonald, W. H., Medina, M., Meijer, H. J., Nordberg, E. K., Maclean, D. J., Ospina-Giraldo, M. D., Morris, P. F., Phuntumart, V., Putnam, N. H., Rash, S., Rose, J. K., Sakihama, Y., Salamov, A. A., Savidor, A., Scheuring, C. F., Smith, B. M., Sobral, B. W., Terry, A., Torto-Alalibo, T. A., Win, J., Xu, Z., Zhang, H., Grigoriev, I. V., Rokhsar, D. S., and Boore, J. L. 2006. Phytophthora genome sequences uncover evolutionary origins and mechanisms of pathogenesis. Science 313:1261-1266. 
Van Poucke, K., Franceschini, S., Webber, J. F., Vercauteren, A., Turner, J. A., McCracken, A. R., Heungens, K., and Brasier, C. M. 2012. Discovery of a fourth evolutionary lineage of Phytophthora ramorum: EU2. Fungal Biol. 116:1178-1191.

Vercauteren, A., Boutet, X., D’hondt, L., Van Bockstaele, E., Maes, M., Leus, L., Chandelier, A., and Heungens, K. 2011. Aberrant genome size and instability of Phytophthora ramorum oospore progenies. Fungal Genet. Biol. 48:537-543.

Vij, S., Kuhl, H., Kuznetsova, I. S., Komissarov, A., Yurchenko, A. A., Van Heusden, P., Singh, S., Thevasagayam, N. M., Prakki, S. R., Purushothaman, K., Saju, J. M., Jiang, J., Mbandi, S. K., Jonas, M., Hin Yan Tong, A., Mwangi, S., Lau, D., Ngoh, S. Y., Liew, W. C., Shen, X., Hon, L. S., Drake, J. P., Boitano, M., Hall, R., Chin, C. S., Lachumanan, R., Korlach, J., Trifonov, V., Kabilov, M., Tupikin, A., Green, D., Moxon, S., Garvin, T., Sedlazeck, F. J., Vurture, G. W., Gopalapillai, G., Kumar Katneni, V., Noble, T. H., Scaria, V., Sivasubbu, S., Jerry, D. R., O’Brien, S. J., Schatz, M. C. Dalmay, T., Turner, S. W., Lok, S., Christoffels, A., and Orbán, L. 2016. Chromosomal-level assembly of the Asian seabass genome using long sequence reads and multi-layered scaffolding. PLoS Genet. 12:e1005954.

Walker, B. J., Abeel, T., Shea, T., Priest, M., Abouelliel, A., Sakthikumar, S., Cuomo, C. A., Zeng, Q., Wortman, J., Young, S. K., and Earl, A. M. 2014. Pilon: An integrated tool for comprehensive microbial variant detection and genome assembly improvement. PLoS One 9:e112963.

Wang, Q., Han, C., Ferreira, A. O., Yu, X., Ye, W., Tripathy, S., Kale, S. D., Gu, B., Sheng, Y., Sui, Y., Wang, X., Zhang, Z., Cheng, B., Dong, S., Shan, W., Zheng, X., Dou, D., Tyler, B. M., and Wang, Y. 2011. Transcriptional programming and functional interactions within the Phytophthora sojae RXLR effector repertoire. Plant Cell 23:2064-2086.
Whisson, S. C., Boevink, P. C., Moleleki, L., Avrova, A. O., Morales, J. G., Gilroy, E. M., Armstrong, M. R., Grouffaud, S., van West, P., Chapman, S., Hein, I., Toth, I. K., Pritchard, L., and Birch, P. R. 2007. A translocation signal for delivery of oomycete effector proteins into host plant cells. Nature 450:115-118.

Win, J., Morgan, W., Bos, J., Krasileva, K. V., Cano, L. M., ChaparroGarcia, A., Ammar, R., Staskawicz, B. J., and Kamoun, S. 2007. Adaptive evolution has targeted the C-terminal domain of the RXLR effectors of plant pathogenic oomycetes. Plant Cell 19: 2349-2369.

Winnenburg, R., Baldwin, T. K., Urban, M., Rawlings, C., Köhler, J., and Hammond-Kosack, K. E. 2006. PHI-base: A new database for pathogen host interactions. Nucleic Acids Res. 34:D459-D464.

Yang, Z. 1997. PAML: A program package for phylogenetic analysis by maximum likelihood. Comput. Appl. Biosci. 13:555-556.

Yin, L., An, Y., Qu, J. 2017. Genome sequence of Plasmoparaviticola and insight into the pathogenic mechanism. Sci. Rep. 7:46553.

Yin, L., Li, X., Xiang, J., Qu, J., Zhang, Y., Dry, I. B., and Lu, J. 2015. Characterization of the secretome of Plasmopara viticola by de novo transcriptome analysis. Physiol. Mol. Plant P. 91:1-10.

Yin, Y., Mao, X., Yang, J., Chen, X., Mao, F., and Xu, Y. 2012. dbCAN: A web resource for automated carbohydrate-active enzyme annotation. Nucleic Acids Res. 40:W445-W451.

Zhang, M., Zhang, Y., Scheuring, C. F., Wu, C. C., Dong, J. J., and Zhang, H. B. 2012. Preparation of megabase-sized DNA from a variety of organisms using the nuclei method for advanced genomics research. Nat. Protoc. 7:467-478. 